\title{
BOUNDEDNESS OF MULTILINEAR PSEUDO-DIFFERENTIAL OPERATORS ON MODULATION SPACES
}

\author{
SHAHLA MOLAHAJLOO, KASSO A. OKOUDJOU, AND GÖTZ E. PFANDER
}

\begin{abstract}
Boundedness results for multilinear pseudodifferential operators on products of modulation spaces are derived based on ordered integrability conditions on the short-time Fourier transform of the operators' symbols. The flexibility and strength of the introduced methods is demonstrated by their application to the bilinear and trilinear Hilbert transform.
\end{abstract}

\section{INTRODUCTION AND MOTIVATION}

Pseudodifferential operators have long been studied in the context of partial differential equations [39, 40, 42, 57, 59, 67, 69]. Among the most investigated topics on such operators are minimal smoothness and decay conditions on their symbols that guarantee their boundedness on function spaces of interest. In recent years, results from time-frequency analysis have been exploited to obtain boundedness results on so-called modulation spaces, which in turn yield boundedness on Bessel potential spaces, Sobolev spaces, and Lebesgue spaces via well established embedding results. In this paper, we develop time-frequency analysis based methods in order to establish boundedness of classes multilinear pseudodifferential operators on products of modulation spaces.

1.1. Pseudodifferential operators. A pseudodiffrential operator is an operator $T_{\sigma}$ formally defined through its symbol $\sigma$ by

$$
T_{\sigma} f(x)=\int_{\mathbb{R}^{d}} \sigma(x, \xi) \hat{f}(\xi) e^{2 \pi i x \cdot \xi} d \xi
$$

where the Fourier transformation is formally given by $(\mathcal{F} f)(\xi)=\widehat{f}(\xi)=\int_{\mathbb{R}^{d}} e^{-2 \pi i x \cdot \xi} f(x) d x$. Hörmander symbol classes are arguably the most used in investigating pseudodifferential operators. In particular, the class of smooth symbols with bounded derivatives was shown to yield bounded operator on $L^{2}$ in the celebrated work of Calderón and Vaillancourt [11]. More specifically, if $\sigma \in S_{0,0}^{0}$, that is, for all non-negative integers $\alpha, \beta$ there exists $C_{\alpha, \beta}$ with

$$
\left|\partial_{x}^{\alpha} \partial_{\xi}^{\beta} \sigma(x, \xi)\right| \leq C_{\alpha, \beta},
$$

then $T_{\sigma}$ maps $L^{2}$ into itself.

1.2. Time-frequency analysis of pseudodifferential operators. In [55], J. Sjöstrand defined a class of bounded operators on $L^{2}$ whose symbols do not have to satisfy a differentiability assumption and which contains those operators with symbol in $S_{0,0}^{0}$. He proved that this class of symbols forms an algebra under the so-called twisted convolution [30, 34, 55, 56. Incidentally, symbols of Sjöstrand's class operators are characterized by their membership in the modulation space $M^{\infty, 1}$, a space of tempered distributions introduced by Feitchinger via integrability and decay conditions on the distributions' short-time Fourier transform [20]. Gröchenig and Heil

Date: March 26, 2022.

2000 Mathematics Subject Classification. Primary 47G30; Secondary 35S99, 42A45, 42B15, 42 B35. 
then significantly extended Sjöstrands results by establishing the boundedness of his pseudodifferential operators on all modulation spaces [35.

These and similar results on pseudodifferential operators were recently extended by Molahajloo and Pfander through the introduction of ordered integrability conditions on the short-time Fourier transform of the operators' symbols [49]. Similar approaches have been used to derive other boundedness results of pseudodifferential operators on modulation space like spaces [10]. The approach of varying integration orders of short-time Fourier transforms of, here, symbols of multilinear operators lies at the center of this paper.

Today, the functional analytical tools developed to analyze pseudodifferential operators on modulation spaces form an integral part of time-frequency analysis. They are used, for example, to model time-varying filters prevalent in signal processing. By now, a robust body of work stemming from this point of view has been developed [18, 35, 36, 37, 54, 60, 61, 63, 66, and has lead to a number of applications to areas such as seismic imaging, and communication theory [47, 58.

1.3. Multilinear pseudodifferential operators. A multilinear pseudo-differential operator $T_{\sigma}$ with distributional symbol $\sigma$ on $\mathbb{R}^{(m+1) d}$, is formally given by

$$
\left(T_{\sigma} \boldsymbol{f}\right)(x)=\int_{\mathbb{R}^{m d}} e^{2 \pi i x \cdot\left(\sum_{i=1}^{d} \xi_{i}\right)} \sigma(x, \boldsymbol{\xi}) \widehat{f}_{1}\left(\xi_{1}\right) \widehat{f}_{2}\left(\xi_{2}\right) \ldots \widehat{f_{m}}\left(\xi_{m}\right) d \boldsymbol{\xi} .
$$

Here and in the following we use boldface characters as $\boldsymbol{\xi}=\left(\xi_{1}, \ldots, \xi_{m}\right)$ to denote products of $m$ vectors $\xi_{i} \in \mathbb{R}^{d}$, and it will not cause confusion to use the symbol $\boldsymbol{f}$ for both, a vector of $m$ functions or distributions $\boldsymbol{f}=\left(f_{1}, \ldots, f_{m}\right)$, that is, a vector valued function or distribution on $\mathbb{R}^{d}$, and the rank one tensor $\boldsymbol{f}=f_{1} \otimes \ldots \otimes f_{m}$, a function or distribution on $\mathbb{R}^{m d}$. For example, we write $\widehat{\boldsymbol{f}}(\boldsymbol{\xi})=\widehat{f_{1}}\left(\xi_{1}\right) \cdot \ldots \cdot \widehat{f_{m}}\left(\xi_{m}\right)$, while $\widehat{\boldsymbol{f}}(\xi)=\left(\widehat{f_{1}}(\xi), \ldots, \widehat{f_{m}}(\xi)\right)$.

A trivial example of a multilinear operator is given by the constant symbol $\sigma \equiv 1$. Clearly, $T_{\sigma}(\boldsymbol{f})$ is simply the product $f_{1}(x) f_{2}(x) \ldots f_{m}(x)$. Thus, Hölder's inequality determines boundedness on products of Lebesgue spaces. On the other hand, when the symbol is independent of the space variable $x$, that is, when $\sigma(x, \boldsymbol{\xi}) \equiv \tau(\boldsymbol{\xi})$, the $T_{\sigma}=T_{\tau}$ is a multilinear Fourier multipliers. We refer to [2, 3, 17, 32, 48, 50] and the references therein for a small sample of the vast literature on multilinear pseudodiffrential operators.

One of the questions that has been repeatedly investigated relates to (minimal) conditions on the symbols $\sigma$ that would guarantee the boundedness of (1.2) on products of certain function spaces, see [17, Theorem 34]. For example, one can ask if a multilinear version of (1.1) exist. Bényi and Torres ([2]) proved that unless additional conditions are added, there exist symbols which satisfy such multilinear estimates but for which the corresponding multilinear pseudodifferential operators are unbounded on products of certain Lebesgue spaces. Indeed, in the bilinear case, that is, when $m=2$, the class of operators whose symbols satisfy for all non-negative integers $\alpha, \beta, \gamma$,

$$
\left|\partial_{x}^{\alpha} \partial_{\xi}^{\beta} \partial_{\eta}^{\gamma} \sigma(x, \xi, \eta)\right| \leq C_{\alpha, \beta, \gamma}
$$

contains operators that do not map $L^{2} \times L^{2}$ into $L^{1}$.

Multilinear pseudodifferential operators in the context of their boundedness on modulation spaces, were first investigated in [6, 7]. Results obtained in this setting have been used to establish well posedness for a number of non-linear PDEs in these spaces [5, 9]. For example, and as opposed to the classical analysis of multilinear pseudodifferential operators, it was proved in [7] that symbols satisfying (1.3) yield boundedness from $L^{2} \times L^{2}$ into the modulation space $M^{1, \infty}$, a space that contains $L^{1}$. The current paper offers some new insights and results in this line of investigation. 
1.4. Our contributions. Modulation spaces are defined by imposing integrability conditions on the short-time Fourier transform of the distribution at hand. Following ideas from Molahajloo and Pfander [49, we impose various ordered integrability conditions on the short-time Fourier transform of a tempered distribution $\sigma$ on $\mathbb{R}^{(m+1) d}$ which is a symbol of a multilinear pseudodifferential operator. By using this new setting, we establish new boundedness results for multilinear pseudodifferential operators on products of modulations spaces. For example, the following result follows from our main result, Theorem 4.1 .

Theorem 1.1. If $1 \leq p_{0}, p_{1}, p_{2}, q_{1}, q_{2}, q_{3} \leq \infty$ satisfy

$$
\frac{1}{p_{0}} \leq \frac{1}{p_{1}}+\frac{1}{p_{2}} \text { and } \quad 1+\frac{1}{q_{3}} \leq \frac{1}{q_{1}}+\frac{1}{q_{2}}
$$

and if for some Schwartz class function $\varphi$, the symbol short-time Fourier transform

$\left.\mathcal{V}_{\varphi} \sigma\left(x, t_{1}, t_{2}, \xi_{1}, \xi_{2}, \nu\right)=\iiint \sigma\left(\widetilde{x}, \widetilde{\xi_{1}}, \widetilde{\xi_{2}}\right) \varphi(x-\widetilde{x}) \varphi\left(\xi_{1}-\widetilde{\xi}_{1}\right) \varphi\left(\xi_{2}-\widetilde{\xi}_{2}\right)\right) e^{-2 \pi i\left(x \nu-t_{1} \xi_{1}-t_{2} \xi_{2}\right)} d \widetilde{x} d \widetilde{\xi_{1}} d \widetilde{\xi_{2}}$ satisfies

$$
\|\sigma\|_{\mathcal{M}^{(\infty, 1,1) ;(\infty, \infty, 1)}}=\int \sup _{\xi_{1}, \xi_{2}} \iint \sup _{x}\left|\mathcal{V}_{\varphi} \sigma\left(x, t_{1}, t_{2}, \xi_{1}, \xi_{2}, \nu\right)\right| d t_{1} d t_{2} d \nu<\infty
$$

then the pseudodifferential operator $T_{\sigma}$ initially defined on $S\left(\mathbb{R}^{d}\right) \times S\left(\mathbb{R}^{d}\right)$ by

$$
T_{\sigma}\left(f_{1}, f_{2}\right)(x)=\iint e^{2 \pi i x \cdot\left(\xi_{1}+\xi_{2}\right)} \sigma\left(x, \xi_{1}, \xi_{2}\right) \widehat{f}_{1}\left(\xi_{1}\right) \widehat{f}_{2}\left(\xi_{2}\right) d \xi_{2} d \xi_{1}
$$

extends to a bounded bilinear operator from $M^{p_{1}, q_{1}} \times M^{p_{2}, q_{2}}$ into $M^{p_{0}, q_{3}}$. Moreover, there exists a constant $C>0$ that only depends on $d$, the $p_{i}$, and $q_{i}$ with

$$
\left\|T_{\sigma}\left(f_{1}, f_{2}\right)\right\|_{M^{p_{0}, q_{3}}} \leq C\|\sigma\|_{\mathcal{M}^{(\infty, 1,1) ;(\infty, \infty, 1)}}\left\|f_{1}\right\|_{M^{p_{1}, q_{1}}}\left\|f_{2}\right\|_{M^{p_{2}, q_{2}}} .
$$

We note that the classical modulation space $M^{\infty, 1}\left(\mathbb{R}^{3 d}\right)$ can be continuously embedded into $\mathcal{M}^{(\infty, 1,1),(\infty, \infty, 1)}\left(\mathbb{R}^{3 d}\right)$ implicitly defined by (1.4). Indeed,

$$
\begin{aligned}
\|\sigma\|_{\mathcal{M}^{(\infty, 1,1) ;(\infty, \infty, 1)}} & =\int \sup _{\xi_{1}, \xi_{2}} \iint \sup _{x}\left|\mathcal{V}_{\varphi} \sigma\left(x, t_{1}, t_{2}, \xi_{1}, \xi_{2}, \nu\right)\right| d t_{1} d t_{2} d \nu \\
& \leq \iiint \sup _{x, \xi_{1}, \xi_{2}}\left|\mathcal{V}_{\varphi} \sigma\left(x, t_{1}, t_{2}, \xi_{1}, \xi_{2}, \nu\right)\right| d t_{1} d t_{2} d \nu=\|\sigma\|_{M^{\infty ; 1}} .
\end{aligned}
$$

As a consequence Theorem 1.1 already extends the main result, Theorem 3.1, in [7].

The herein presented new approach allows us to investigate the boundedness of the bilinear Hilbert transform on products of modulation spaces. Indeed, in the one dimensional setting, $d=1$, it can be shown that the symbol of the bilinear Hilbert transform

$$
\sigma_{H} \in \mathcal{M}^{(\infty, 1, r) ;(\infty, \infty, 1)} \backslash \mathcal{M}^{(\infty, 1,1) ;(\infty, \infty, 1)}
$$

for all $r>1$. Hence, $\sigma_{H} \notin M^{\infty, 1}$ and existing methods to investigate multilinear pseudodifferential operators on products of modulations spaces are not applicable. Using the techniques developed below, we obtain novel and wide reaching boundedness results for the bilinear Hilbert transform on the product of modulation spaces. For example, as a special case of our result, we prove that the bilinear Hilbert transform is bounded from $L^{2} \times L^{2}$ into the modulation space $M^{1+\epsilon, 1}$ for any $\epsilon>0$.

The results established here aim at generality and differ in technique from the ground breaking results about the bilinear Hilbert transformed as obtained by Lacey and Thiele [44, 43, 45, 46]. They are therefore not easily compared to those obtained using "hard analysis" techniques. Nonetheless, using our results and some embeddings of modulation spaces into Lebesgue space, 
we discuss the relation of our results on the boundedness of the bilinear Hilbert transform to the known classical results.

The herein given framework is flexible enough to allow an initial investigation of the trilinear Hilbert transform. Here we did not try to optimize our results but just show through some examples how one can tackle this more difficult operator in the context of modulation spaces.

1.5. Outline. We introduce our new class of symbols based on a modification of the shorttime Fourier transform in Section 2. We then prove a number of technical results including some Young-type inequalities, that form the foundation of our main results. Section 3 contains most of the key results needed to establish our results. This naturally leads to our main results concerning the boundedness of multilinear pseudodifferential operators on product of modulation spaces. Section 4 is devoted to applications of our results. In Section 4.1 we specialize our results to the bilinear case, proving boundedness results of bilinear pseudodifferential operators on products of modulation spaces. We then consider as example the bilinear Hilbert transform in Section 4.2. In Section 4.3 we initiate an investigation of the boundedness of the trilinear Hilbert transform on products of modulation spaces.

\section{Symbol Classes for multilinear PSEUdodifferential operators}

2.1. Background on modulation spaces. Let $\mathbf{r}=\left(r_{1}, r_{2}, \ldots, r_{m}\right)$ where $1 \leq r_{i}<\infty, i=$ $1,2, \ldots, m$. The mixed norm space $L^{r}\left(\mathbb{R}^{m d}\right)$ is Banach space of measurable functions $F$ on $\mathbb{R}^{m d}$ with finite norm [1]

$$
\|F\|_{L^{\mathbf{r}}}=\left(\int_{\mathbb{R}^{d}} \ldots\left(\int_{\mathbb{R}^{d}}\left(\int_{\mathbb{R}^{d}}\left|F\left(x_{1}, \ldots, x_{m}\right)\right|^{r_{1}} d x_{1}\right)^{r_{2} / r_{1}} d x_{2} \ldots\right)^{r_{m} / r_{m-1}} d x_{m}\right)^{1 / r_{m}} .
$$

Similarly, we define $L^{\mathbf{r}}\left(\mathbb{R}^{m d}\right)$ where $r_{i}=\infty$ for some indices $i$. For a nonnegative measurable function $w$ on $\mathbb{R}^{m d}$ wee define $L_{w}^{\mathbf{r}}\left(\mathbb{R}^{m d}\right)$ to be the space all $F$ on $\mathbb{R}^{m d}$ for which $F w$ is in $L^{\mathbf{r}}\left(\mathbb{R}^{m d}\right)$, that is, $\|F\|_{L_{w}^{\mathbf{r}}}=\|F w\|_{L^{\mathbf{r}}}<\infty$.

For the purpose of this paper, we define a mixed norm space depending on a permutation that determines the order of integration. For a permutation $\rho$ on $\{1,2, \ldots, n\}$, the weighted mixed norm space $L_{w}^{\boldsymbol{r} ; \rho}\left(\mathbb{R}^{m d}\right)$ is the set of all measurable functions $F$ on $\mathbb{R}^{m d}$ for which

$$
\begin{gathered}
\|F\|_{L_{w}^{r ; \rho}}=\left(\int _ { \mathbb { R } ^ { d } } \left(\int _ { \mathbb { R } ^ { d } } \left(\ldots \left(\int_{\mathbb{R}^{d}}\left|F\left(x_{1}, x_{2}, \ldots, x_{n}\right) w\left(x_{1}, x_{2}, \ldots, x_{n}\right)\right|^{r_{\rho(1)}}\right.\right.\right.\right. \\
\left.\left.\left.d x_{\rho(1)}\right)^{r_{\rho(2)} / r_{\rho(1)}} d x_{\rho(2)}\right)^{r_{\rho(3)} / r_{\rho(2)}} \ldots d x_{\rho(n)}\right)^{1 / r_{\rho(n)}}
\end{gathered}
$$

is finite.

Let $M_{\nu}$ denote modulation by $\nu \in \mathbb{R}^{d}$, namely, $M_{\nu} f(x)=e^{2 \pi i t \cdot \nu} f(x)$, and let $T_{t}$ be translation by $t \in \mathbb{R}^{d}$, that is, $T_{t} f(x)=f(x-t)$. The short-time Fourier transform $V_{\phi} f$ of $f \in \mathcal{S}^{\prime}\left(\mathbb{R}^{d}\right)$ with respect to the Gaussian window $\phi(x)=e^{-\|x\|^{2}}$ is given by

$$
V_{\phi} f(t, \nu)=\mathcal{F}\left(f T_{t} \phi\right)(\nu)=\left(f, M_{\nu} T_{t} \phi\right)=\int f(x) e^{-2 \pi i x \nu} \phi(x-t) d x .
$$

The modulation space $M^{p, q}\left(\mathbb{R}^{d}\right), 1 \leq p, q \leq \infty$, is a Banach space consisting of those $f \in \mathcal{S}^{\prime}\left(\mathbb{R}^{d}\right)$ with

$$
\|f\|_{M^{p, q}}=\left\|V_{\phi} f\right\|_{L^{p, q}}=\left(\int\left(\int\left|V_{\phi} f(t, \nu)\right|^{p} d t\right)^{q / p} d \nu\right)^{1 / q}<\infty,
$$

with usual adjustment of the mixed norm space if $p=\infty$ and/or $q=\infty$. We refer to [20, 34] for background on modulation spaces. 
In the sequel we consider weight functions $w$ on $\mathbb{R}^{2(m+1) d}$. We assume that $w$ is continuous and sub-multiplicative, that is, $w(x+y) \leq C w(x) w(y)$. Associated to $w$ will be a family of $w$ moderate weight functions $v$. That is $v$ is positive, continuous and satisfies $v(x+y) \leq C w(x) v(y)$.

2.2. A new class of symbols. The commonly used short-time Fourier transform analyzes functions in timet; as symbols have time and frequency variables, we base the herein used shorttime Fourier transform on a Fourier transform that takes Fourier transforms in time variables and inverse Fourier transforms in frequency variables. We then order the variables, first time, then frequency. That is, we follow the idea of symplectic Fourier transforms $\mathcal{F}_{s}$ on phase space,

$$
\mathcal{F}_{s} F(\boldsymbol{t}, \nu)=\iint_{\mathbb{R}^{(m+1) d}} F(x, \boldsymbol{\xi}) e^{2 \pi i(\boldsymbol{\xi} \boldsymbol{t}-x \nu)} d \boldsymbol{\xi} d x .
$$

For $F \in \mathcal{S}^{\prime}\left(\mathbb{R}^{(m+1) d}\right)$ and $\phi \in \mathcal{S}\left(\mathbb{R}^{(m+1) d}\right)$, we define the symbol short-time Fourier transform $\mathcal{V}_{\phi} F$ of $F$ with respect to $\phi$ by

$$
\begin{aligned}
\mathcal{V}_{\phi} F(x, \boldsymbol{t}, \boldsymbol{\xi}, \nu) & =\mathcal{F}_{s}\left(F T_{(x, \boldsymbol{\xi})} \phi\right)(\boldsymbol{t}, \nu)=\left\langle F, M_{(-\nu, t)} T_{(x, \boldsymbol{\xi})} \phi\right\rangle \\
& =\int_{\mathbb{R}^{m d}} \int_{\mathbb{R}^{d}} e^{-2 \pi i(\widetilde{x} \nu-t \widetilde{\boldsymbol{\xi}})} F(\widetilde{x}, \widetilde{\boldsymbol{\xi}},) \phi(\widetilde{x}-x, \widetilde{\boldsymbol{\xi}}-\boldsymbol{\xi}) d \widetilde{x} d \widetilde{\boldsymbol{\xi}}
\end{aligned}
$$

where $x, \nu \in \mathbb{R}^{d}$, and $\boldsymbol{t}, \boldsymbol{\xi} \in \mathbb{R}^{m d}$. Note that the symbol short-time Fourier transform is related to the ordinary short-time Fourier transform by

$$
\mathcal{V}_{\phi} F(x, \boldsymbol{t}, \boldsymbol{\xi}, \nu)=V_{\phi} F(x, \boldsymbol{\xi}, \nu,-\boldsymbol{t}) .
$$

Modulation spaces for symbols of multilinear operators are then defined by requiring the symbol short-time Fourier transform of an operator to be in certain weighted $L^{p}$ spaces. To describe these, we fix decay parameters $1 \leq p_{0}, p_{1}, \ldots, p_{m}, q_{1}, q_{2}, \ldots, q_{m}, q_{m+1} \leq \infty$, and permutations $\kappa$ on $\{0,1, \ldots, m\}$ and $\rho$ on $\{1, \ldots, m, m+1\}$. The latter indicate the integration order of the time, respectively frequency, variables. Put, $\mathbf{p}=\left(p_{1}, p_{2}, \ldots, p_{m}\right), \mathbf{q}=\left(q_{1}, q_{2}, \ldots, q_{m}\right)$ and let $w$ be a weight function on $\mathbb{R}^{2(m+1) d}$. Then $L_{w}^{\left(p_{0}, \mathbf{p}\right), \kappa ;\left(\mathbf{q}, q_{m+1}\right), \rho}\left(\mathbb{R}^{2(m+1) d}\right)$ is the mixed norm space consisting of those measurable functions $F$ for which the norm

$$
\begin{aligned}
& \|F\|_{L_{w}^{\left(p_{0}, \mathbf{p}\right), \kappa ;\left(\mathbf{q}, q_{m+1}\right), \rho}}=\left(\int _ { \mathbb { R } ^ { d } } \left(\int _ { \mathbb { R } ^ { d } } \left(\ldots \left(\int _ { \mathbb { R } ^ { d } } \left(\int _ { \mathbb { R } ^ { d } } \left(\ldots \left(\int _ { \mathbb { R } ^ { d } } \left(\int_{\mathbb{R}^{d}}\right.\right.\right.\right.\right.\right.\right.\right. \\
& \quad\left|w\left(t_{0}, t_{1}, \ldots, t_{m}, \xi_{1}, \ldots, \xi_{m}, \xi_{m+1}\right) F\left(t_{0}, t_{1}, \ldots, t_{m}, \xi_{1}, \ldots, \xi_{m}, \xi_{m+1}\right)\right|^{p_{\kappa(0)}} \\
& \left.\left.\left.\left.\left.\quad d t_{\kappa(0)}\right)^{p_{\kappa(1)} / p_{\kappa(0)}} d t_{\kappa(1)}\right)^{p_{\kappa(2)} / p_{\kappa(1)}} \ldots d t_{\kappa(m)}\right)^{q_{\rho(1)} / p_{\kappa(m)}} d \xi_{\rho(1)}\right)^{q_{\rho(2)} / q_{\rho(1)}} \ldots d \xi_{\rho(m+1)}\right)^{1 / q_{\rho(m+1)}}
\end{aligned}
$$

is finite. The weighted symbol modulation space $\mathcal{M}_{w}^{\left(p_{0}, \mathbf{p}\right), \kappa ;\left(\mathbf{q}, q_{m+1}\right), \rho}\left(\mathbb{R}^{(m+1) d}\right)$ is composed of those $F \in \mathcal{S}^{\prime}\left(\mathbb{R}^{(m+1) d}\right)$ with

$$
\|F\|_{\mathcal{M}_{w}^{\left(p_{0}, \mathbf{p}\right), \kappa ;\left(\mathbf{q}, q_{m+1}\right), \rho}}=\left\|\mathcal{V}_{\phi} F\right\|_{L_{w}^{\left(p_{0}, \mathbf{p}\right), \kappa ;\left(\mathbf{q}, q_{m+1}\right), \rho}}<\infty .
$$

When $\kappa$ and $\rho$ are identity permutations, then we denote $L_{w}^{\left(p_{0}, \mathbf{p}\right), \kappa ;\left(\mathbf{q}, q_{m+1}\right), \rho}\left(\mathbb{R}^{2(m+1) d}\right)$ and $\mathcal{M}_{w}^{\left(p_{0}, \mathbf{p}\right), \kappa ;\left(\mathbf{q}, q_{m+1}\right), \rho}\left(\mathbb{R}^{2(m+1) d}\right)$ by $L_{w}^{\left(p_{0}, \mathbf{p}\right) ;\left(\mathbf{q}, q_{0}\right)}\left(\mathbb{R}^{2(m+1) d}\right)$ and $\mathcal{M}_{w}^{\left(p_{0}, \mathbf{p}\right) ;\left(\mathbf{q}, q_{0}\right)}\left(\mathbb{R}^{2(m+1) d}\right)$, respectively. The dependence of the norm on the choice of $\kappa, \rho$, as well as the advantage of choosing a particular order will be discussed in Section 2.4.

\footnotetext{
${ }^{1}$ For clarity, we always refer to the variables $x, y, t$ as time variables, even though a physical interpretation of time necessitates $d=1$. Alternatively, one can consider multivariate $x, y, t$ as spatial variables.
} 
For simplicity of notation, we set $S(\boldsymbol{\xi})=\sum_{i=1}^{m} \xi_{i}$. For functions $g$ and components of $\boldsymbol{f}$ in $\mathcal{S}\left(\mathbb{R}^{d}\right)$, the Rihaczek transform $R(\boldsymbol{f}, g)$ of $\boldsymbol{f}$ and $g$ is defined by

$$
R(\boldsymbol{f}, g)(x, \boldsymbol{\xi})=e^{2 \pi i x \cdot\left(\xi_{1}+\ldots+\xi_{m}\right)} \widehat{f}_{1}\left(\xi_{1}\right) \cdot \ldots \cdot \widehat{f}_{m}\left(\xi_{m}\right) \overline{g(x)}=e^{2 \pi i x \cdot S(\boldsymbol{\xi})} \widehat{\boldsymbol{f}}(\boldsymbol{\xi}) \overline{g(x)} .
$$

Multilinear pseudo-differential operators are related to Rihaczek transforms by

$$
\left\langle T_{\sigma} \boldsymbol{f}, g\right\rangle=\langle\sigma, \overline{R(\boldsymbol{f}, g)}\rangle
$$

a-priori for all functions $f_{i}$ and $g$ in $\mathcal{S}\left(\mathbb{R}^{d}\right)$ and symbols $\sigma \in \mathcal{S}\left(\mathbb{R}^{(m+1) d}\right)$.

With $x \pm \boldsymbol{t}=x \pm\left(t_{1}, \ldots, t_{m}\right)=\left(x \pm t_{1}, \ldots, x \pm t_{m}\right)$, it can be easily seen that

$$
R(\boldsymbol{f}, g)(x, \boldsymbol{\xi})=\mathcal{F}_{\boldsymbol{t} \rightarrow \boldsymbol{\xi}}(\boldsymbol{f}(\cdot+x)) \bar{g}(x)
$$

where

$$
\mathcal{F}_{\boldsymbol{t} \rightarrow \boldsymbol{\xi}}(\boldsymbol{f}(\cdot+x))(\boldsymbol{\xi})=\int_{\mathbb{R}^{m d}} e^{-2 \pi i \boldsymbol{t} \cdot \boldsymbol{\xi}} \boldsymbol{f}(\boldsymbol{t}+x) d \boldsymbol{t}
$$

Lemma 2.1. For $\varphi$ real-valued, $\varphi=(\varphi, \ldots, \varphi), \boldsymbol{f}=\left(f_{1}, f_{2}, \ldots, f_{m}\right) \in \mathcal{S}\left(\mathbb{R}^{d}\right)^{m}$, and $g \in \mathcal{S}\left(\mathbb{R}^{d}\right)$,

$V_{T_{A}(\boldsymbol{\varphi} \otimes \varphi)} T_{A}(\overline{\boldsymbol{f}} \otimes g)(x,-\boldsymbol{\xi}, \boldsymbol{t}, \nu)=\overline{V_{\varphi} f_{1}\left(x-t_{1}, \xi_{1}\right) \ldots V_{\varphi} f_{m}\left(x-t_{m}, \xi_{m}\right)} \cdot V_{\varphi} g(x, \nu-S(\boldsymbol{\xi}))$.

Moreover,

$$
\left(V_{\overline{R(\boldsymbol{\varphi}, \varphi)}} \overline{R(\boldsymbol{f}, g)}\right)(x, \boldsymbol{\xi}, \nu, \boldsymbol{t})=e^{-2 \pi i \boldsymbol{\xi} \boldsymbol{t}}\left(\mathcal{V}_{T_{A}(\boldsymbol{\varphi} \otimes \varphi)} T_{A}(\overline{\boldsymbol{f}} \otimes g)\right)(x,-\boldsymbol{t}, \nu, \boldsymbol{\xi}),
$$

and in particular,

$$
\left|\left(V_{\overline{R(\boldsymbol{\varphi}, \varphi)}} \overline{R(\boldsymbol{f}, g)}\right)(x, \boldsymbol{\xi}, \nu, \boldsymbol{t})\right|=\left|V_{T_{A}(\boldsymbol{\varphi} \otimes \varphi)} T_{A}(\overline{\boldsymbol{f}} \otimes g)(x,-\boldsymbol{\xi},-\boldsymbol{t}, \nu)\right|
$$

Proof. We compute

$$
\begin{aligned}
& \left(V_{T_{A}(\boldsymbol{\varphi} \otimes \varphi)} T_{A}(\overline{\boldsymbol{f}} \otimes g)\right)(x,-\boldsymbol{\xi}, \boldsymbol{t}, \nu) \\
& =\int_{\mathbb{R}^{m d}} \int_{\mathbb{R}^{d}} e^{-2 \pi i(\widetilde{x} \nu+\widetilde{\boldsymbol{t} \xi})} T_{A}(\overline{\boldsymbol{f}} \otimes g)(\widetilde{x}, \widetilde{\boldsymbol{t}}) T_{A}(\boldsymbol{\varphi} \otimes \varphi)(\widetilde{x}-x, \widetilde{\boldsymbol{t}}-\boldsymbol{t}) d \widetilde{x} d \widetilde{\boldsymbol{t}} \\
& =\int_{\mathbb{R}^{d}}\left(\int_{\mathbb{R}^{m d}} e^{-2 \pi i \widetilde{\boldsymbol{t} \xi}} \overline{\boldsymbol{f}}(\widetilde{x}-\widetilde{\boldsymbol{t}}) \boldsymbol{\varphi}(\widetilde{x}-x-\widetilde{\boldsymbol{t}}+\boldsymbol{t}) d \widetilde{\boldsymbol{t}}\right) e^{-2 \pi i \widetilde{x} \nu} g(\widetilde{x}) \varphi(\widetilde{x}-x) d \widetilde{x} \\
& =\int_{\mathbb{R}^{d}} \int_{\mathbb{R}^{m d}} \overline{\boldsymbol{f}}(\boldsymbol{s}) g(\widetilde{x}) e^{-2 \pi i(\nu \widetilde{x}+\boldsymbol{\xi}(\widetilde{x}-\boldsymbol{s}))} \boldsymbol{\varphi}(\boldsymbol{s}-(x-\boldsymbol{t})) \varphi(\widetilde{x}-x) d \widetilde{x} d \boldsymbol{s} \\
& =\left\{\sqrt{\int_{\mathbb{R}^{m d}} e^{-2 \pi i \boldsymbol{\xi} \boldsymbol{f}} \boldsymbol{f}(\boldsymbol{s}) \boldsymbol{\varphi}(\boldsymbol{s}-(x-\boldsymbol{t})) d \boldsymbol{s}}\right\}\left\{\int_{\mathbb{R}^{d}} e^{-2 \pi i(\nu+S(\boldsymbol{\xi})) \widetilde{x}} g(\widetilde{x}) \varphi(\widetilde{x}-x) d \widetilde{x}\right\} \\
& =\overline{\left(V_{\varphi} \boldsymbol{f}\right)(x-\boldsymbol{t}, \boldsymbol{\xi})}\left(V_{\varphi} g\right)(x, \nu+S(\boldsymbol{\xi})) .
\end{aligned}
$$

Further,

$$
\begin{aligned}
& \left(V_{\overline{R(\boldsymbol{\varphi}, \varphi)}} \overline{R(\boldsymbol{f}, g)}\right)(x, \boldsymbol{\xi}, \nu, \boldsymbol{t}) \\
= & \int_{\mathbb{R}^{m d}} \int_{\mathbb{R}^{d}} e^{-2 \pi i(\nu \widetilde{x}+\boldsymbol{t} \widetilde{\boldsymbol{\xi}})} \overline{R(\boldsymbol{f}, g)(\widetilde{x}, \widetilde{\boldsymbol{\xi}})} R(\boldsymbol{\varphi}, \varphi)(\widetilde{x}-x, \widetilde{\boldsymbol{\xi}}-\boldsymbol{\xi}) d \widetilde{x} d \widetilde{\boldsymbol{\xi}} \\
= & \int_{\mathbb{R}^{m d}} \int_{\mathbb{R}^{d}} e^{-2 \pi i(\nu \widetilde{x}+\boldsymbol{t} \widetilde{\boldsymbol{\xi}})} \mathcal{F}_{\widetilde{\boldsymbol{t}} \rightarrow \widetilde{\boldsymbol{\xi}}}(\overline{\boldsymbol{f}}(\widetilde{x}-\cdot)) g(\widetilde{x}) \overline{\mathcal{F}_{\widetilde{\boldsymbol{t}} \rightarrow \widetilde{\boldsymbol{\xi}}-\xi}(\boldsymbol{\varphi}(\widetilde{x}-x-\cdot))} \varphi(\widetilde{x}-x) d \widetilde{x} d \widetilde{\boldsymbol{\xi}} \\
= & \int_{\mathbb{R}^{m d}} \int_{\mathbb{R}^{d}} e^{-2 \pi i(\nu \widetilde{x}+\boldsymbol{t} \widetilde{\boldsymbol{\xi}})} \mathcal{F}_{\widetilde{\boldsymbol{t}} \rightarrow \widetilde{\boldsymbol{\xi}}}(\overline{\boldsymbol{f}}(\widetilde{x}-\cdot)) g(\widetilde{x}) \mathcal{F}_{\widetilde{\boldsymbol{t}} \rightarrow \boldsymbol{\xi}-\widetilde{\boldsymbol{\xi}}}(\boldsymbol{\varphi}(\widetilde{x}-x-\cdot)) \varphi(\widetilde{x}-x) d \widetilde{x} d \widetilde{\boldsymbol{\xi}}
\end{aligned}
$$


On the other hand, by using Parseval identity we have

$$
\begin{aligned}
& \left(V_{T_{A}(\boldsymbol{\varphi} \otimes \varphi)} T_{A}(\overline{\boldsymbol{f}} \otimes g)\right)(x, \boldsymbol{t}, \nu, \boldsymbol{\xi}) \\
= & \int_{\mathbb{R}^{d}} \int_{\mathbb{R}^{m d}} e^{-2 \pi i(\widetilde{x} \nu+\widetilde{\boldsymbol{t} \xi})} T_{A}(\overline{\boldsymbol{f}} \otimes g)(\widetilde{x}, \widetilde{\boldsymbol{t}}) T_{A}(\boldsymbol{\varphi} \otimes \varphi)(\widetilde{x}-x, \widetilde{\boldsymbol{t}}-\boldsymbol{t}) d \widetilde{x} d \widetilde{\boldsymbol{t}} \\
= & \int_{\mathbb{R}^{d}}\left(\int_{\mathbb{R}^{m d}} e^{-2 \pi i \widetilde{\boldsymbol{t}} \boldsymbol{f}} \overline{\boldsymbol{f}}(\widetilde{x}-\widetilde{\boldsymbol{t}}) \boldsymbol{\varphi}(\widetilde{x}-x-\widetilde{\boldsymbol{t}}+\boldsymbol{t}) d \widetilde{\boldsymbol{t}}\right) e^{-2 \pi i \widetilde{x} \nu} g(\widetilde{x}) \varphi(\widetilde{x}-x) d \widetilde{x} \\
= & \int_{\mathbb{R}^{d}} \int_{\mathbb{R}^{m d}} \mathcal{F}_{\widetilde{\boldsymbol{t}} \rightarrow \widetilde{\boldsymbol{\xi}}}(\overline{\boldsymbol{f}}(\widetilde{x}-\cdot)) \mathcal{F}_{\widetilde{\boldsymbol{t}} \rightarrow \widetilde{\boldsymbol{\xi}}}^{-1}\left(e^{-2 \pi i \widetilde{\boldsymbol{\xi}}} \boldsymbol{\varphi}(\widetilde{x}-x+\boldsymbol{t}-\cdot)\right) e^{-2 \pi i \widetilde{x} \nu} g(\widetilde{x}) \varphi(\widetilde{x}-x) d \widetilde{\boldsymbol{\xi}} d \widetilde{x} .
\end{aligned}
$$

But,

$$
\mathcal{F}_{\widetilde{\boldsymbol{t}} \rightarrow \widetilde{\boldsymbol{\xi}}}^{-1}\left(e^{-2 \pi i \widetilde{\boldsymbol{\xi}}} \boldsymbol{\varphi}(\widetilde{x}-x+\boldsymbol{t}-\cdot)\right)=e^{-2 \pi i \boldsymbol{t}(\boldsymbol{\xi}-\widetilde{\boldsymbol{\xi}})} \mathcal{F}_{\boldsymbol{\gamma} \rightarrow \boldsymbol{\xi}-\widetilde{\boldsymbol{\xi}}}(\boldsymbol{\varphi}(\widetilde{x}-x-\cdot)),
$$

therefore,

$$
\begin{aligned}
& \left(V_{T_{A}(\boldsymbol{\varphi} \otimes \varphi)} T_{A}(\overline{\boldsymbol{f}} \otimes g)\right)(x, \boldsymbol{t}, \nu, \boldsymbol{\xi})= \\
& e^{-2 \pi i t \boldsymbol{\xi}} \int_{\mathbb{R}^{m d}} \int_{\mathbb{R}^{d}} e^{2 \pi i(\boldsymbol{t} \widetilde{\boldsymbol{\xi}}-v \widetilde{x})} \mathcal{F}_{\widetilde{\boldsymbol{t}} \rightarrow \widetilde{\boldsymbol{\xi}}}(\overline{\boldsymbol{f}}(\widetilde{x}-\cdot)) \mathcal{F}_{\widetilde{\boldsymbol{t}} \rightarrow \boldsymbol{\xi}-\widetilde{\boldsymbol{\xi}}}(\varphi(\widetilde{x}-x-\cdot)) g(\widetilde{x}) \varphi(\widetilde{x}-x) d \widetilde{x} d \widetilde{\boldsymbol{\xi}}
\end{aligned}
$$

2.3. Young type results. The following results are consequences of Young's inequality and will be central in proving our main results. We use the convention that summation over the empty set is equal to 0 .

Lemma 2.2. Suppose that $1 \leq p_{k}, r_{k} \leq \infty$ for $k=0,1, \ldots, m$ and

(A1) $p_{k} \leq r_{k}, k=1, \ldots, m$;

(A2) $\sum_{\ell=1}^{k} \frac{1}{p_{\ell}}-\frac{1}{r_{\ell}} \leq \frac{1}{r_{0}}-\frac{1}{p_{k+1}}, \quad k=0, \ldots, m-1$;

(A3) $\sum_{\ell=1}^{m} \frac{1}{p_{\ell}}-\frac{1}{r_{\ell}}=\frac{1}{r_{0}}-\frac{1}{p_{0}}$;

then $F(x, \boldsymbol{t})=\boldsymbol{f}(x-\boldsymbol{t}) g(x)$ satisfies

$$
\|F\|_{L^{\left(r_{0}, r\right)}} \leq\|g\|_{L^{p_{0}}}\|\boldsymbol{f}\|_{L^{\boldsymbol{p}}}
$$

Proof. For simplicity, we use capital letters for the reciprocals of $p_{k}, r_{k}$, that is, $P_{k}=1 / p_{k}$, $R_{k}=1 / r_{k}, k=0, \ldots, m$. Recalling that summation over the empty set is defined as 0 , our assumptions (A1) - (A3) are simply

(A1) $P_{k} \geq R_{k}, k=1, \ldots, m$;

(A2) $R_{0}-P_{k+1} \geq \sum_{\ell=1}^{k} P_{\ell}-R_{\ell}, \quad k=0, \ldots, m-1$;

(A3) $\sum_{\ell=0}^{m} R_{\ell}=\sum_{\ell=0}^{m} P_{\ell}$.

Define $1 / b_{1}=B_{1}=R_{0}+R_{1}-P_{1}$, and for $k=2, \ldots, m$,

$$
\begin{aligned}
1 / b_{k}=B_{k} & =B_{k-1}+R_{k}-P_{k} \\
& =R_{0}+\sum_{\ell=1}^{k} R_{\ell}-P_{\ell} .
\end{aligned}
$$


The first application of Young's inequality below requires that

$$
p_{1} / r_{0}, r_{1} / r_{0}, b_{1} / r_{0} \geq 1 \text { and } 1 /\left(p_{1} / r_{0}\right)+1 /\left(b_{1} / r_{0}\right)=1+1 /\left(r_{1} / r_{0}\right) .
$$

This translates to $R_{0} \geq R_{1}, B_{1}, P_{1}$ and $P_{1}+B_{1}=R_{0}+R_{1}$ which is equivalent to

$$
R_{0} \geq R_{1}, P_{1}, R_{0}+R_{1}-P_{1} .
$$

But, condition (A1) of the hypothesis implies that $P_{1} \geq R_{1}$. Thus we have, $R_{0} \geq R_{1}, P_{1}$ and $P_{1} \geq R_{1}$, that is, $R_{0} \geq P_{1} \geq R_{1}$. Similarly, the successive applications of Young's inequality follow by replacing $p_{1}, r_{1}, b_{1}, r_{0}$ by $p_{k}, r_{k}, b_{k}, b_{k-1}$, respectively. That is, we require

$$
B_{k-1} \geq R_{k}, B_{k-1}+R_{k}-P_{k}, P_{k}
$$

which is equivalent to $B_{k-1} \geq P_{k} \geq R_{k}$ which follows from (A1).

We shall also use the standard fact that for $0<\alpha, \beta, \gamma, \delta<\infty$,

$$
\left\||f|^{\alpha}\right\|_{L^{\beta}}^{\gamma}=\left\||f|^{\alpha \delta}\right\|_{L^{\beta / \delta}}^{\frac{\gamma}{\delta}}
$$

and set $\widetilde{f}(x)=f(-x)$. We compute

$$
\begin{aligned}
& \|F\|_{L^{r_{0}, r}}^{r_{m}} \\
& =\int_{\mathbb{R}^{d}}\left(\int_{\mathbb{R}^{d}} \ldots\left(\int_{\mathbb{R}^{d}}\left(\int_{\mathbb{R}^{d}}\left|f_{1}\left(x-t_{1}\right) \ldots f_{m}\left(x-t_{m}\right) g(x)\right|^{r_{0}} d x\right)^{\frac{r_{1}}{r_{0}}} d t_{1}\right)^{\frac{r_{2}}{r_{1}}} \ldots\right)^{r_{m}} d t_{m} \\
& =\int_{\mathbb{R}^{d}}\left(\int_{\mathbb{R}^{d}} \ldots\left(\int_{\mathbb{R}^{d}}\left(\int_{\mathbb{R}^{d}}\left|\tilde{f}_{1}\left(t_{1}-x\right)\left(T_{t_{2}} \tilde{f}_{2}(x) \ldots T_{t_{m}} \tilde{f}_{m}(x) g(x)\right)\right|^{r_{0}} d x\right)^{\frac{r_{1}}{r_{0}}} d t_{1}\right)^{\frac{r_{2}}{r_{1}}} \ldots\right)^{r_{m}} d t_{m} \\
& =\int_{\mathbb{R}^{d}}\left(\int_{\mathbb{R}^{d}} \ldots\left(\int_{\mathbb{R}^{d}}\left(\left|\tilde{f}_{1}\right|^{r_{0}} *\left|T_{t_{2}} \tilde{f}_{2} \ldots T_{t_{m}} \tilde{f}_{m} g\right|^{r_{0}}\left(t_{1}\right)\right)^{\frac{r_{1}}{r_{0}}} d t_{1}\right)^{\frac{r_{2}}{r_{1}}} \ldots\right)^{r_{m}} d t_{m} \\
& =\int_{\mathbb{R}^{d}}\left(\int_{\mathbb{R}^{d}} \ldots\left(\int_{\mathbb{R}^{d}}\left\|\left|\tilde{f}_{1}\right|^{r_{0}} *\left|T_{t_{2}} \widetilde{f}_{2} \ldots T_{t_{m}} \tilde{f}_{m} g\right|^{r_{0}}\right\|_{L^{r_{1} / r_{0}}}^{\frac{r_{1}}{r_{1}} \frac{r_{2}}{r_{1}}} d t_{2}\right)^{\frac{r_{3}}{r_{2}}} \ldots\right)^{r_{m}} d t_{m} \\
& \leq \int_{\mathbb{R}^{d}}\left(\int_{\mathbb{R}^{d}} \ldots\left(\int_{\mathbb{R}^{d}}\left\|\left|\widetilde{f}_{1}\right|^{r_{0}}\right\|_{L^{p_{1} / r_{0}}}^{\frac{r_{2}}{r_{0}}}\left\|\left|T_{t_{2}} \widetilde{f}_{2} \ldots T_{t_{m}} \widetilde{f}_{m} g\right|^{r_{0}}\right\|_{L^{b_{1} / r_{0}}}^{\frac{r_{2}}{r_{0}}} d t_{2}\right)^{\frac{r_{3}}{r_{2}}} \ldots\right)^{r_{m}} d t_{m} \\
& =\int_{\mathbb{R}^{d}}\left(\int_{\mathbb{R}^{d}} \ldots\left(\int_{\mathbb{R}^{d}}\left\|\tilde{f}_{1}\right\|_{L^{p_{1}}}^{r_{2}}\left\|\left.T_{t_{2}} f_{2} \ldots T_{t_{m}} f_{m} g\right|^{b_{1}}\right\|_{L^{1}}^{\frac{r_{2}}{b_{1}}} d t_{2}\right)^{\frac{r_{3}}{r_{2}}} \ldots\right)^{r_{m}} d t_{m} \\
& =\left\|f_{1}\right\|_{L^{p_{1}}}^{r_{m}} \int_{\mathbb{R}^{d}}\left(\int_{\mathbb{R}^{d}} \ldots\left(\int_{\mathbb{R}^{d}}\left(\int_{\mathbb{R}^{d}}\left|f_{2}\left(x-t_{2}\right) \ldots f_{m}\left(x-t_{m}\right) g(x)\right|^{b_{1}} d x\right)^{\frac{r_{2}}{b_{1}}} d t_{2}\right)^{\frac{r_{3}}{r_{2}}} \ldots\right)^{r_{m}} d t_{m} \\
& \text {... } \\
& \leq\left\|f_{1}\right\|_{L^{p_{1}}}^{r_{m}} \ldots\left\|f_{m-1}\right\|_{L^{p_{m-1}}}^{r_{m}} \int_{\mathbb{R}^{d}}\left(\int_{\mathbb{R}^{d}}\left|f_{m}\left(x-t_{m}\right) g(x)\right|^{b_{m-1}} d x\right)^{\frac{r_{m}}{b_{m-1}}} d t_{m} \\
& =\left\|f_{1}\right\|_{L^{p_{1}}}^{r_{m}} \ldots\left\|f_{m-1}\right\|_{L^{p_{m-1}}}^{r_{m}}\left\|\left|\widetilde{f}_{m}\right|^{b_{m-1}} *|g|^{b_{m-1}}\right\|_{L^{\frac{r_{m}}{b_{m-1}}}}^{\frac{r_{m}}{b_{m}}} \\
& \leq\left\|f_{1}\right\|_{L^{p_{1}}}^{r_{m}} \cdots\left\|f_{m-1}\right\|_{L^{p_{m-1}}}^{r_{m}}\left\|f_{m}\right\|_{L^{p_{m}}}^{r_{m}}\left\||g|^{p_{0}}\right\|_{L^{1}}^{\frac{r_{m}}{p_{0}}} \\
& =\left\|f_{1}\right\|_{L^{p_{1}}}^{r_{m}} \ldots\left\|f_{m}\right\|_{L^{p_{m}}}^{r_{m}}\|g\|_{L^{p_{0}}}^{r_{m}}
\end{aligned}
$$

where each inequality stems from an application of Young's inequality for convolutions. In the final step, we used $b_{m}=p_{0}$ which follows by combining the definition of $b_{m}$ with hypothesis (A3). 
Remark 2.3. Observe that if we would add the condition $p_{0} \leq r_{0}$ in hypothesis (A1) of Lemma 2.2, then (A1) and (A3) would combine to imply $p_{k}=r_{k}$ for $k=0, \ldots, m$. Indeed, the strength of Lemma 2.2 lies in the fact that $p_{0} \leq r_{0}$ and $p_{k}=r_{k}$ for $k=0, \ldots, m$ are not implied by the hypotheses. Setting $\Delta_{k}=\frac{1}{p_{k}}-\frac{1}{r_{k}}$ for $k=0, \ldots, m$, (A1) in Lemma 2.2 is $\Delta_{1}, \ldots, \Delta_{m} \geq 0$ and condition (A3) becomes $\Delta_{0}+\sum_{k=1}^{m} \Delta_{k}=0$, a condition that allows $\Delta_{0}$ to be negative, that is $p_{0}>r_{0}$. In short, all $\Delta_{k}>0$ contribute to compensate for $\Delta_{0}=r_{0}-p_{0}$ being negative.

Let us now briefly discuss condition (A2) in Lemma 2.2. For $k=0$, we have $0 \leq \frac{1}{r_{0}}-\frac{1}{p_{1}}$. To satisfy condition (A2) for $k=1$, we increase the left hand side by $\Delta_{1}=\frac{1}{p_{1}}-\frac{1}{r_{1}} \geq 0$, add to the right hand side the possibly negative term $\frac{1}{p_{1}}-\frac{1}{p_{2}}$, and require that the sum on the left remains bounded above by the sum on the right. For $k=2$, we increase the left hand side by $\Delta_{2}=\frac{1}{p_{2}}-\frac{1}{r_{2}} \geq 0$ and add to the right hand side $\frac{1}{p_{2}}-\frac{1}{p_{3}}$, maintaining that the right hand side dominates the left hand side. This is illustrated in Figure 1 below.

In the case $m=1$, the conditions $\Delta_{1} \geq 0$ and $\Delta_{0}+\Delta_{1}=0$ from Lemma 2.2 are amended by the requirement $r_{0} \leq p_{1}$, and, for example, if $r_{0}=1, p_{0}=2$, then Lemma 2.2 is applicable whenever $1 \geq \frac{1}{p_{1}}=\frac{1}{r_{1}}+\frac{1}{2}$, that is, if $1 \leq p_{1}=\frac{2 r_{1}}{r_{1}+2}$.

If $m=2$, then $\Delta_{1}, \Delta_{2} \geq 0$ and $\Delta_{0}+\Delta_{1}+\Delta_{2}=0$ from Lemma 2.2 are combined with the condition $r_{0} \leq p_{1}$ and $\Delta_{1} \leq \frac{1}{r_{0}}-\frac{1}{p_{2}}$. It is crucial in what follows to observe that these conditions are sensitive to the order of the $p_{k}$ and the $r_{k}$. For example, the parameters $r_{0}=1, p_{0}=2$, $r_{1}=1=p_{1}, p_{2}=1, r_{2}=2$ satisfy the hypothesis, while $r_{0}=1, p_{0}=2, r_{2}=1=p_{2}, p_{1}=1$, $r_{1}=2$ do not.

Indeed, if for some $k, \Delta_{k}=\frac{1}{p_{k}}-\frac{1}{r_{k}}$ is much smaller than $\frac{1}{p_{k}}-\frac{1}{p_{k+1}}$, then we would profit more from this if $k$ is a small index, that is, the respective summands play a role early on in the summation.

Below, we shall use this idea and reorder the indices. This allows us to first choose $\kappa(1)=$ $k_{1} \in\{1, \ldots, d\}$ with $\Delta_{\kappa(1)}=\frac{1}{p_{\kappa(1)}}-\frac{1}{r_{\kappa(1)}}$ small, and then $\kappa(2)=k_{2}$ so that $\frac{1}{p_{\kappa(1)}}-\frac{1}{p_{\kappa(2)}}$ is large. Clearly, the feasibility of $\kappa(2)$ also depends on the size of $\Delta_{\kappa(2)}=\frac{1}{p_{\kappa(2)}}-\frac{1}{r_{\kappa(2)}}$, so finding an optimal order cannot be achieved with a greedy algorithm. Moreover, note that the spaces $\mathcal{M}^{\left(p_{0}, \mathbf{p}\right), \kappa ;\left(\mathbf{q}, \mathbf{q}_{\mathbf{m}+\mathbf{1}}\right), \sigma}$ and $\mathcal{M}^{\left(p_{0}, \mathbf{p}\right), \text { id; }\left(\mathbf{q}, \mathbf{q}_{\mathbf{m}+\mathbf{1}}\right) \text {,id }}$ are not identical, hence, we cannot choose $\kappa$ and $\rho$ arbitrarily.

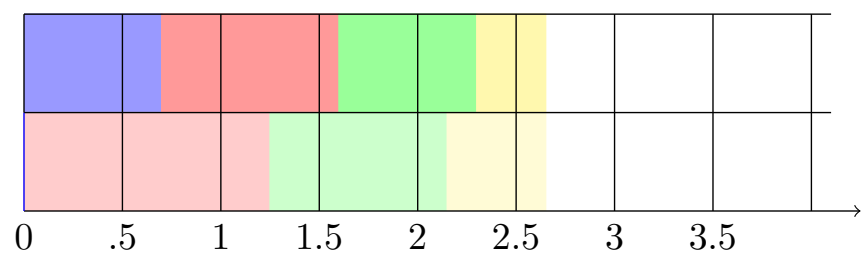

Figure 1. Depiction of condition (A2) in Lemma 2.2. After adding a pair of colored fields, the top row must always exceed the lower row, with the lower row finally catching up in the last step, see Remark 2.3 .

Remark 2.4. Note that conditions (A1) and (A3) follow from (but are not equivalent to) the simpler condition

(A4) $1 \leq r_{0} \leq p_{1} \leq r_{1} \leq p_{2} \leq \ldots \leq r_{m-1} \leq p_{m} \leq r_{m} \leq \infty$.

Equality (A3) can then be satisfied by choosing an appropriate $p_{0} \geq 1$.

The inequalities in (A1) imply that the LHS of (A2) is positive and, hence, always $r_{0} \leq p_{k} \leq r_{k}$ for all $k$. Also, (A1) and (A2) necessitate $p_{k} \leq r_{k} \leq p_{m+1}$. 
Similarly to Lemma 2.2, we show the following.

Lemma 2.5. Suppose that $1 \leq q_{k}, s_{k} \leq \infty$ for $k=1, \ldots, m+1$ and

(B1) $q_{k} \geq s_{k}, k=1, \ldots, m$;

(B2) $\sum_{\ell=k+1}^{m} \frac{1}{q_{\ell}}-\frac{1}{s_{\ell}} \geq \frac{1}{s_{m+1}}-\frac{1}{q_{k}}, \quad k=1, \ldots, m$;

(B3) $\sum_{\ell=1}^{m} \frac{1}{q_{\ell}}-\frac{1}{s_{\ell}}=\frac{1}{s_{m+1}}-\frac{1}{q_{m+1}}$;

then for $G(\boldsymbol{t}, x)=\boldsymbol{f}(\boldsymbol{t}) g(x+S(\boldsymbol{t}))$ we have

$$
\|G\|_{L^{s, s_{m+1}}} \leq\|\boldsymbol{f}\|_{L^{q}}\|g\|_{L^{q_{m+1}}} .
$$

Proof. As before, our computations involve the introduction of an auxiliary parameter $b_{k}$. We start with a formal computation, namely,

$$
\begin{aligned}
& \|G\|_{L^{r, s_{m+1}}}^{s_{m+1}} \\
& =\int_{\mathbb{R}^{d}}\left(\int_{\mathbb{R}^{d}} \ldots\left(\int_{\mathbb{R}^{d}}\left|f_{1}\left(t_{1}\right) \ldots f_{m}\left(t_{m}\right) g\left(x+t_{1}+\ldots+t_{m}\right)\right|^{s_{1}} d t_{1}\right)^{\frac{s_{2}}{s_{1}}} \ldots d t_{m}\right)^{\frac{s_{m+1}}{s_{m}}} d x \\
& =\int_{\mathbb{R}^{d}}\left(\int_{\mathbb{R}^{d}}\left|\widetilde{f}_{m}\left(t_{m}\right)\right|^{s_{m}}\left(\ldots \int_{\mathbb{R}^{d}}\left|\widetilde{f}_{2}\left(t_{2}\right)\right|^{s_{2}}\left(\int_{\mathbb{R}^{d}}\left|\widetilde{f}_{1}\left(t_{1}\right) g\left(x-t_{1}-t_{2}-\ldots-t_{m}\right)\right|^{s_{1}} d t_{1}\right)^{\frac{s_{2}}{s_{1}}} d t_{2}\right)^{\frac{s_{3}}{s_{2}}} \ldots\right)^{\frac{s_{m+1}}{s_{m}}} d x \\
& \left.=\int_{\mathbb{R}^{d}}\left(\int_{\mathbb{R}^{d}}\left|\tilde{f}_{m}\left(t_{m}\right)\right|^{s_{m}}\left(\ldots \int_{\mathbb{R}^{d}}\left|\widetilde{f}_{2}\left(t_{2}\right)\right|^{s_{2}}\left(\left|\tilde{f}_{1}\right|^{s_{1}} *|g|^{s_{1}}\left(x-t_{2}-t_{3}-\ldots-t_{m}\right)\right)^{\frac{s_{2}}{s_{1}}} d t_{2}\right)^{\frac{s_{3}}{s_{2}}} d t_{3}\right)^{\frac{s_{4}}{s_{3}}} \ldots\right)^{\frac{s_{m}+1}{s_{m}}} d x \\
& \text {... } \\
& =\int_{\mathbb{R}^{d}}\left(\left|\tilde{f}_{m}\right|^{s_{m}} *\left(\left|\tilde{f}_{m-1}\right|^{s_{m-1}} *\left(\ldots\left(\left|\tilde{f}_{2}\right|^{s_{2}} *\left(\left|\tilde{f}_{1}\right|^{s_{1}} *|g(x)|^{s_{1}}\right)^{\frac{s_{2}}{s_{1}}}\right)^{\frac{s_{3}}{s_{2}}} \ldots\right)^{\frac{s_{m}}{s_{m}-1}}\right)^{\frac{s_{m+1}}{s_{m}}} d x\right. \\
& =\|\left|\widetilde{f}_{m}\right|^{s_{m}} *\left(\left|\widetilde{f}_{m-1}\right|^{s_{m-1}} *\left(\ldots\left(\left|\tilde{f}_{2}\right|^{s_{2}} *\left(\left|\tilde{f}_{1}\right|^{s_{1}} *|g|^{s_{1}}\right)^{\frac{s_{2}}{s_{1}}}\right)^{\frac{s_{3}}{s_{2}}} \ldots\right)^{\frac{s_{m}}{s_{m-1}}} \|_{L^{\frac{s_{m+1}}{s_{m}}}}^{\frac{s_{m}}{s_{m}}}\right. \\
& \leq\left\|\left|\widetilde{f}_{m}\right|^{s_{m}}\right\|_{L^{q_{m} / s_{m}}}^{\frac{s_{m+1}}{s_{m}}} \|\left(\left|\widetilde{f}_{m-1}\right|^{s_{m-1}} *\left(\ldots\left(\left|\widetilde{f}_{2}\right|^{s_{2}} *\left(\left|\widetilde{f}_{1}\right|^{s_{1}} *|g|^{s_{1}}\right)^{\frac{s_{2}}{s_{1}}}\right)^{\frac{s_{3}}{s_{2}}} \ldots\right)^{\frac{s_{m}}{s_{m}-1}} \|_{L^{\frac{s_{m}+1}{b_{m}} / s_{m}}}^{\frac{s_{m}}{s^{2}}}\right. \\
& =\left\|f_{m}\right\|_{L^{q_{m}}}^{s_{m+1}}\left\|\left|\tilde{f}_{m-1}\right|^{s_{m-1}} *\left(\ldots\left(\left|\tilde{f}_{2}\right|^{s_{2}} *\left(\left|\tilde{f}_{1}\right|^{s_{1}} *|g|^{s_{1}}\right)^{\frac{s_{2}}{s_{1}}}\right)^{\frac{s_{3}}{s_{2}}} \ldots\right)^{\frac{s_{m-1}}{s_{m-2}}}\right\|_{L^{b_{m} / s_{m-1}}}^{\frac{s_{m+1}}{s_{m}-1}} \\
& =\left\|f_{m}\right\|_{L^{q_{m}}}^{s_{m+1}} \ldots\left\|f_{2}\right\|_{L^{q_{2}}}^{s_{m+1}}\left\|\left|\tilde{f}_{1}\right|^{s_{1}} *|g|^{s_{1}}\right\|_{L^{b_{2} / s_{1}}}^{\frac{s_{m+1}}{s_{1}}} \\
& \leq\left\|f_{m}\right\|_{L^{q_{m}}}^{s_{m+1}} \cdots\left\|f_{2}\right\|_{L^{q_{2}}}^{s_{m+1}}\left\|\left|\tilde{f}_{1}\right|^{s_{1}}\right\|_{L^{q_{1} / s_{1}}}^{\frac{s_{m+1}}{s_{1}}}\left\||g|^{s_{1}}\right\|_{L^{q_{m+1} / s_{1}}}^{\frac{s_{m+1}}{s_{1}}} \\
& =\left\|f_{m}\right\|_{L^{q_{m}}}^{s_{m+1}} \ldots\left\|f_{1}\right\|_{L^{q_{1}}}^{s_{m+1}}\|g\|_{L^{q_{m+1}}}^{s_{m+1}} \text {. }
\end{aligned}
$$

To justify the first application of Young's inequality, we require

$$
\frac{1}{\frac{q_{m}}{s_{m}}}+\frac{1}{\frac{b_{m}}{s_{m}}}=1+\frac{1}{\frac{s_{m+1}}{s_{m}}}, \quad \frac{q_{m}}{s_{m}}, \frac{b_{m}}{s_{m}}, \frac{s_{m+1}}{s_{m}} \geq 1 .
$$

Using reciprocals, this is equivalent to

$$
Q_{m}+B_{m}=S_{m}+S_{m+1}, \quad S_{m} \geq Q_{m}, B_{m}, S_{m+1},
$$

that is,

$$
B_{m}=S_{m}-Q_{m}+S_{m+1}, \quad S_{m} \geq Q_{m}, B_{m}, S_{m+1}
$$


The subsequent application of Young's inequality requires

$$
\frac{1}{\frac{q_{m-1}}{s_{m-1}}}+\frac{1}{\frac{b_{m-1}}{s_{m-1}}}=1+\frac{1}{\frac{b_{m}}{s_{m-1}}}, \quad \frac{q_{m-1}}{s_{m-1}}, \frac{b_{m-1}}{s_{m-1}}, \frac{b_{m}}{s_{m-1}} \geq 1 .
$$

Using reciprocals, this is equivalent to

$$
B_{m-1}=S_{m-1}-Q_{m-1}+B_{m}=S_{m+1}+\sum_{\ell=m-1}^{m} S_{\ell}-Q_{\ell}, \quad S_{m-1} \geq Q_{m-1}, B_{m-1}, B_{m} .
$$

In general, for $k=1, \ldots, m-2$, we require

$$
B_{m-k}=S_{m-k}-Q_{m-k}+B_{m-k+1}=S_{m+1}+\sum_{\ell=m-k}^{m} S_{\ell}-Q_{\ell}, \quad S_{m-k} \geq Q_{m-k}, B_{m-k}, B_{m-k+1},
$$

and finally, for the last application of Young's inequality, we require

$$
Q_{m+1}=S_{1}-Q_{1}+B_{2}=S_{m+1}+\sum_{\ell=m-k}^{m} S_{\ell}-Q_{\ell}, \quad S_{1} \geq Q_{1}, Q_{m+1}, B_{2} .
$$

Now, $S_{k} \geq Q_{k}$ for $k=1, \ldots, m$ implies

$$
0 \leq S_{m+1} \leq B_{m} \leq B_{m-1} \leq \ldots \leq B_{3} \leq B_{2} \leq Q_{m+1}
$$

hence, it suffices to postulate aside of $S_{k} \geq Q_{k}$ for $k=1, \ldots, m$ the conditions $S_{k} \geq B_{k}$ for $k=2, \ldots, m$ and $S_{1} \geq Q_{m+1}, B_{2}$. For $k=2, \ldots, m$, we use that $\sum_{\ell=1}^{m+1} S_{\ell}-Q_{\ell}=0$ implies $\sum_{\ell=k}^{m} S_{\ell}-Q_{\ell}=-S_{m+1}+Q_{m+1}-\sum_{\ell=1}^{k-1} S_{\ell}-Q_{\ell}$ in order to rewrite $S_{k} \geq B_{k}$ in form of

$$
S_{k} \geq B_{k}=S_{m+1}+\sum_{\ell=k}^{m} S_{\ell}-Q_{\ell}=Q_{m+1}-\sum_{\ell=1}^{k-1} S_{\ell}-Q_{\ell}
$$

which is

$$
Q_{m+1}-S_{k} \leq \sum_{\ell=1}^{k-1} S_{\ell}-Q_{\ell}
$$

For $k=1$, the above covers the condition $Q_{m+1} \leq S_{1}$.

In summary, for $k=1, \ldots, m+1$ we obtained the sufficient conditions

(B1') $q_{k} \geq s_{k}, k=1, \ldots, m$

$$
\begin{aligned}
& \text { (B2') } \sum_{\ell=1}^{k} \frac{1}{s_{\ell}}-\frac{1}{q_{\ell}} \geq \frac{1}{q_{m+1}}-\frac{1}{s_{k+1}}, \quad k=0, \ldots, m-1 ; \\
& \text { (B3') } \sum_{\ell=1}^{m} \frac{1}{s_{\ell}}-\frac{1}{q_{\ell}}=\frac{1}{q_{m+1}}-\frac{1}{s_{m+1}} .
\end{aligned}
$$

Forming the difference of (B3') and (B2') gives

$$
\text { (B2") } \sum_{\ell=k+1}^{m} \frac{1}{s_{\ell}}-\frac{1}{q_{\ell}} \leq \frac{1}{s_{k+1}}-\frac{1}{s_{m+1}}, \quad k=0, \ldots, m-1 .
$$

Reindexing leads to

$$
\text { (B2") } \sum_{\ell=k}^{m} \frac{1}{s_{\ell}}-\frac{1}{q_{\ell}} \leq \frac{1}{s_{k}}-\frac{1}{s_{m+1}}, \quad k=1, \ldots, m,
$$

and adding $\frac{1}{q_{k}}-\frac{1}{s_{k}}$ to both sides, and then multiplying both sides by -1 gives 
(B2) $\sum_{\ell=k+1}^{m} \frac{1}{q_{\ell}}-\frac{1}{s_{\ell}} \geq \frac{1}{s_{m+1}}-\frac{1}{q_{k}}, \quad k=1, \ldots, m$.

Remark 2.6. The conditions (B1)-(B3) are similar to those in (A1)-(A3). Indeed, a change of variable $k \rightarrow m+1-k$, that is, renaming $q_{k}=\widetilde{q}_{m+1-k}$ and $s_{k}=\widetilde{s}_{m+1-k}, k=1, \ldots, m+1$, turns (B2) into

$$
\sum_{\ell=k+1}^{m} \frac{1}{\widetilde{q}_{m+1-\ell}}-\frac{1}{\widetilde{s}_{m+1-\ell}} \geq \frac{1}{\widetilde{s}_{m+1-(m+1)}}-\frac{1}{\widetilde{q}_{m+1-k}}=\frac{1}{\widetilde{s}_{0}}-\frac{1}{\widetilde{q}_{m+1-k}}, \quad k=1, \ldots, m .
$$

We have

$$
\sum_{\ell=k+1}^{m} \frac{1}{\widetilde{q}_{m+1-\ell}}-\frac{1}{\widetilde{s}_{m+1-\ell}}=\sum_{\ell^{\prime}=1}^{m-k} \frac{1}{\widetilde{q}_{\ell^{\prime}}}-\frac{1}{\widetilde{s}_{\ell^{\prime}}},
$$

hence, we obtain for $k^{\prime}=m-k$ the conditions

$$
\sum_{\ell^{\prime}=1}^{k^{\prime}} \frac{1}{\widetilde{q}_{\ell^{\prime}}}-\frac{1}{\widetilde{s}_{\ell^{\prime}}} \geq \frac{1}{\widetilde{s}_{0}}-\frac{1}{\widetilde{q}_{k^{\prime}+1}}, \quad k^{\prime}=0, \ldots, m-1 .
$$

We conclude that difference between the conditions in Lemma 2.2 and in Lemma 2.5 lies aside of naming the decay parameters - simply in replacing $\leq$ in (A1) and (A2) by $\geq$ in (B1) and (B2). Hence, it comes to no surprise that (B1) and (B2) follow from, but are not equivalent to

(B4) $1 \leq s_{1} \leq q_{1} \leq s_{2} \leq \ldots \leq q_{m-1} \leq s_{m} \leq q_{m} \leq s_{m+1} \leq \infty$.

Moreover, (B1) implies $\sum_{\ell=k+1}^{m} \frac{1}{q_{\ell}}-\frac{1}{s_{\ell}} \leq 0$, and, hence, $q_{m+1} \geq q_{k}$ for $k=1, \ldots, m$.

2.4. Young type results with permutations. As observed in Remark 2.3, condition (A2) in Lemma 2.2 and, similarly, (B2) in Lemma 2.5 are sensitive to the order of the $p_{k}, r_{k}, q_{k}$, and $s_{k}$.

To obtain a bound for operators as desired, we may have to reorder the parameters. This motivates the introduction of permutations $\kappa$ and $\rho$. In addition to the flexibility obtained at cost of notational complexity, we observe that the permutation of the integration order will allow us to pull out integration with respect to some variables. In fact, setting $t_{0}=x$ and choosing $j=\kappa^{-1}(0)$, we arrive at

$$
\begin{aligned}
& \|F\|_{L^{\left(r_{0}, \boldsymbol{r}\right) ; \kappa}}^{r_{\kappa(m)}} \\
& =\int_{\mathbb{R}^{d}}\left(\int_{\mathbb{R}^{d}} \ldots\left(\int_{\mathbb{R}^{d}}\left(\int_{\mathbb{R}^{d}}\left|f_{1}\left(t_{0}-t_{1}\right) \ldots f_{m}\left(t_{0}-t_{m}\right) g\left(t_{0}\right)\right|^{r_{\kappa(0)}} d t_{\kappa(0)}\right)^{\frac{r_{\kappa(1)}}{r_{\kappa(0)}}} d t_{\kappa(1)}\right)^{\frac{r_{\kappa(2)}}{r_{\kappa(1)}}} \ldots\right)^{r_{\kappa(m)}} d t_{\kappa(m)} \\
& =\int_{\mathbb{R}^{d}}\left(\int_{\mathbb{R}^{d}} \ldots\left(\int_{\mathbb{R}^{d}}\left(\int_{\mathbb{R}^{d}} \prod_{\ell=0}\left|f_{\kappa(\ell)}\left(t_{0}-t_{\kappa(\ell)}\right) g\left(t_{0}\right)\right|^{r_{\kappa(0)}} d t_{\kappa(0)}\right)^{\frac{r_{\kappa(1)}}{r_{\kappa(0)}}} d t_{\kappa(1)}\right)^{\frac{r_{\kappa(2)}}{r_{\kappa(1)}}} \ldots\right)^{r_{\kappa(m)}} d t_{\kappa(m)} \\
& =\left\|f_{\kappa(0)}\right\|_{L^{p_{\kappa(0}(0)}}^{r_{(m)}}\left\|f_{\kappa(1)}\right\|_{L^{p_{\kappa(1)}}}^{r_{\kappa(m)}} \ldots\left\|f_{\kappa(j-1)}\right\|_{L^{p_{\kappa(j-1)}}}^{r_{\kappa(m)} \times} \\
& \int\left(\int \ldots\left(\int\left(\int\left|f_{\kappa(j+1)}\left(x-t_{\kappa(j+1)}\right) \ldots f_{\kappa(m)}\left(x-t_{\kappa(m)}\right) g(x)\right|^{r_{\kappa(j)}} d x\right)^{\frac{r_{\kappa(j+1)}}{r_{\kappa(j)}}} d t_{\kappa(j+1)}\right)^{\left.\frac{r_{\kappa(j+2)}}{r_{\kappa(j+1)}} \ldots\right)^{r_{\kappa(m)}} d t_{\kappa(m)} .}\right.
\end{aligned}
$$

We can then apply Lemma 2.2 to the iterated integral on the right hand side.

This observation leads us to the following result. 
Lemma 2.7. Let $\kappa$ be a permutation on $\{0,1, \ldots, m\}, z=\kappa^{-1}(0)$, and let $1 \leq p_{k}, r_{k} \leq \infty$, $k=0,1, \ldots, m$, satisfy

(A0) $p_{\kappa(\ell)}=r_{\kappa(\ell)}, \ell=0, \ldots, z-1$;

(A1) $p_{\kappa(\ell)} \leq r_{\kappa(\ell)}, \ell=z, \ldots, m$;

(A2) $\sum_{\ell=z+1}^{k} \frac{1}{p_{\kappa(\ell)}}-\frac{1}{r_{\kappa(\ell)}} \leq \frac{1}{r_{0}}-\frac{1}{p_{\kappa(k+1)}}, \quad k=z, \ldots, m-1$;

(A3) $\sum_{\ell=z+1}^{m} \frac{1}{p_{\kappa(\ell)}}-\frac{1}{r_{\kappa(\ell)}}=\frac{1}{r_{0}}-\frac{1}{p_{0}}$.

Then for $F(x, \boldsymbol{t})=\boldsymbol{f}(x-\boldsymbol{t}) g(x)$ it holds

$$
\|F\|_{L^{\left(r_{0}, r\right)_{\kappa}}} \leq\|g\|_{L^{p_{0}}}\|\boldsymbol{f}\|_{L^{p}}
$$

Remark 2.8. Loosely speaking, the decay of a function $F\left(x, t_{1}, \ldots, t_{d}\right)$ in the variables $\left(x, t_{1}, \ldots, t_{d}\right)$ is given by the parameters $\left(p_{0}, p_{1}, \ldots, p_{d}\right)$, that is, $L^{p_{0}}$-decay in $x, L^{p_{1}}$-decay in $t_{1}, \ldots, L^{p_{d} \text {-decay }}$ in $t_{d}$. As we then use the flexibility of order of integration, it is worth noting that Minkowski's inequality for integrals implies that integrating with respect to variables with large exponents last, increases the size of the space.

For example, if $q \geq p$, we have

$$
\|F\|_{L^{(p, q) ;(0,1)}}=\left(\int\left(\int\left|F\left(x, t_{1}\right)\right|^{p} d x\right)^{q / p} d t_{1}\right)^{1 / q} \leq\left(\int\left(\int\left|F\left(x, t_{1}\right)\right|^{q} d x\right)^{p / q} d t_{1}\right)^{1 / p}=\|F\|_{L^{(p, q) ;(1,0)}},
$$

which implies $L^{(p, q) ;(0,1)} \subseteq L^{(p, q) ;(1,0)}$ if $q \geq p$, for example, $L^{(1, \infty) ;(0,1)} \subseteq L^{(1, \infty) ;(1,0)}$. This inclusion is strict in general, for example, choose $F\left(x, t_{1}\right)=g\left(x-t_{1}\right) \in L^{(1, \infty) ;(1,0)} \backslash L^{(1, \infty) ;(0,1)}$ for any function $g \in L^{1}$.

Similarly to Lemma 2.7, we formulate the following.

Lemma 2.9. Let $\rho$ be a permutation on $\{1, \ldots, m+1\}, w=\rho^{-1}(m+1)$, and $1 \leq q_{k}, s_{k} \leq \infty$ be $k=1, \ldots, m+1$ satisfy

(B0) $q_{\kappa(\ell)}=s_{\kappa(\ell)}, \ell=w, \ldots, m$;

(B1) $q_{\rho(k)} \geq s_{\rho(k)}, k=1, \ldots, w-1$;

(B2) $\sum_{\ell=k+1}^{w-1} \frac{1}{q_{\rho(\ell)}}-\frac{1}{s_{\rho(\ell)}} \geq \frac{1}{s_{m+1}}-\frac{1}{q_{\rho(k)}}, \quad k=1, \ldots, w-1$

(B3) $\sum_{\ell=1}^{w-1} \frac{1}{q_{\rho(\ell)}}-\frac{1}{s_{\rho(\ell)}}=\frac{1}{s_{m+1}}-\frac{1}{q_{m+1}}$.

Then $G(\boldsymbol{\xi}, \nu)=\boldsymbol{f}(\boldsymbol{\xi}) g(\nu+S(\boldsymbol{\xi}))$ satisfies

$$
\|G\|_{L^{\left(s, s_{m+1}\right), \rho}} \leq\|\boldsymbol{f}\|_{L^{\boldsymbol{q}}}\|g\|_{L^{q_{m+1}}} .
$$

\section{BOUNDEDNESS ON MODULATION SPACES}

When applying Lemmas 2.2, 2.5, 2.7, and 2.9 in the context of modulation spaces, we can use the property that $M^{p_{1}, q_{1}}$ embeds continuously in $M^{p_{2}, q_{2}}$ if $p_{1} \leq p_{2}$ and $q_{1} \leq q_{2}$. To exploit this in full, the introduction of auxiliary parameters $\widetilde{\boldsymbol{p}}$ and $\widetilde{\boldsymbol{s}}$ is required as illustrated by Example 3.2 below.

Proposition 3.1. Given $1 \leq p_{0}, \boldsymbol{p}, \widetilde{\boldsymbol{p}}, \boldsymbol{q}, \widetilde{\boldsymbol{q}}, q_{m+1}, r_{0}, \boldsymbol{r}, \boldsymbol{s}, s_{m+1} \leq \infty$ with $\boldsymbol{p} \leq \widetilde{\boldsymbol{p}} \leq \boldsymbol{r}$ and $\boldsymbol{s}, \boldsymbol{q} \leq \widetilde{\boldsymbol{q}}$. Let $\kappa$ be a permutation on $\{0, \ldots, m\}$ and let $z=\kappa^{-1}(0)$. Similarly, let $\rho$ be a permutation on $\{1,2, \ldots, m+1\}$ and $w=\rho^{-1}(m+1)$. Assume 

(1) $\sum_{\ell=z+1}^{k} \frac{1}{\widetilde{p}_{\kappa(\ell)}}-\frac{1}{r_{\kappa(\ell)}} \leq \frac{1}{r_{0}}-\frac{1}{\widetilde{p}_{\kappa(k+1)}}, \quad k=z, \ldots, m-1$;
(2) $\sum_{\ell=z+1}^{m} \frac{1}{\widetilde{p}_{\kappa(\ell)}}-\frac{1}{r_{\kappa(\ell)}} \geq \frac{1}{r_{0}}-\frac{1}{p_{0}}$;
(3) $\sum_{\ell=k+1}^{w-1} \frac{1}{\widetilde{q}_{\rho(\ell)}}-\frac{1}{s_{\rho(\ell)}} \geq \frac{1}{s_{m+1}}-\frac{1}{\widetilde{q}_{k}}, \quad k=1, \ldots, w-1$;
(4) $\sum_{\ell=1}^{w-1} \frac{1}{\widetilde{q}_{\rho(\ell)}}-\frac{1}{s_{\rho(\ell)}} \geq \frac{1}{s_{m+1}}-\frac{1}{q_{m+1}}$.

Let $v$ be a weight function on $\mathbb{R}^{2(m+1) d}$ and assume that $w_{0}, w_{1}, \ldots, w_{m}$ are weights on $\mathbb{R}^{2 d}$ such that

$$
v(x, \boldsymbol{t}, \boldsymbol{\xi}, \nu) \leq w_{0}(x, \nu+S(\xi)) w_{1}\left(x-t_{1}, \xi_{1}\right) \cdot \ldots \cdot w_{m}\left(x-t_{m}, \xi_{m}\right) .
$$

For $\varphi \in S\left(\mathbb{R}^{d}\right)$ real valued, $\boldsymbol{f} \in M_{\boldsymbol{w}}^{\boldsymbol{p}, \kappa ; \boldsymbol{q}, \rho}\left(\mathbb{R}^{m d}\right)$, and $g \in M_{w_{0}}^{p_{0}, q_{m+1}}\left(\mathbb{R}^{d}\right)$, we have $\mathcal{V}_{T_{A}(\boldsymbol{\varphi} \otimes \varphi)} T_{A}(\overline{\boldsymbol{f}} \otimes$ $g) \in L_{v}^{\left(r_{0}, \boldsymbol{r}\right)_{\kappa},\left(\boldsymbol{s}, s_{m+1}\right)_{\rho}}\left(\mathbb{R}^{2(m+1) d}\right)$ with

$$
\left\|\mathcal{V}_{T_{A}(\boldsymbol{\varphi} \otimes \varphi)} T_{A}(\overline{\boldsymbol{f}} \otimes g)\right\|_{L_{v}^{\left(r_{0}, \boldsymbol{r}\right), \kappa ;\left(s, s_{m+1}\right), \rho}} \leq C\left\|f_{1}\right\|_{M_{w_{1}}^{p_{1}, q_{1}} \ldots} \ldots f_{m}\left\|_{M_{w_{m}}^{p_{m}, q_{m}}}\right\| g \|_{M_{w_{0}}^{p_{0}, q_{m+1}}},
$$

where the LHS is defined by integrating the variables in the index order

$$
\kappa(0), \kappa(1), \ldots, \kappa(m), \rho(1), \ldots, \rho(m), \rho(m+1) .
$$

In particular,

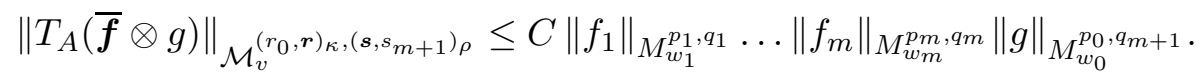

Note that $C$ depends only on the parameters $p_{i}, r_{i}, q_{i}, s_{i}$ and $d$.

Proof. For simplicity we assume $\rho=\kappa=i d$ and use Lemma 2.2 and Lemma 2.5. The general case follows as Lemma 2.7 and Lemma 2.9 followed from Lemma 2.2 and Lemma 2.5.

Let $\boldsymbol{f}=\left(f_{1}, f_{2}, \ldots, f_{m}\right), \boldsymbol{\phi}=(\phi, \phi, \ldots, \phi)$ and $\boldsymbol{w}=\left(w_{1} . w_{2} \ldots w_{m}\right)$. Then

$$
V_{\phi} \boldsymbol{f}=V_{\phi} f_{1} \otimes V_{\phi} f_{2} \otimes \cdots \otimes V_{\phi} f_{m}
$$

and by Lemma 2.1, we have

$$
\mathcal{V}_{T_{A}(\boldsymbol{\varphi} \otimes \varphi)} T_{A}(\overline{\boldsymbol{f}} \otimes g)(x,-\boldsymbol{\xi}, \boldsymbol{t}, \nu)=\overline{V_{\varphi} \boldsymbol{f}(x-\boldsymbol{t}, \boldsymbol{\xi})} V_{\varphi} g(x, \nu-S(\boldsymbol{\xi})),
$$

where $x, \nu \in \mathbb{R}^{d}$, and $\boldsymbol{t}, \boldsymbol{\xi} \in \mathbb{R}^{m d}$.

So, if (3.1) and conditions (2) and (4) above hold with equality, then (A1)-(A3) in Lemma 2.2 and (B1)-(B3) in Lemma 2.5 will hold. Then

$$
\begin{aligned}
& \left\|v(x, \boldsymbol{t}, \boldsymbol{\xi}, \nu) \mathcal{V}_{T_{A}(\boldsymbol{\varphi} \otimes \varphi)} T_{A}(\overline{\boldsymbol{f}} \otimes g)(x, \boldsymbol{t}, \boldsymbol{\xi}, \nu)\right\|_{L^{\left(r_{0}, \boldsymbol{r}\right),\left(\boldsymbol{s}, s_{m+1}\right)}(x, \boldsymbol{t}, \boldsymbol{\xi}, \nu)} \\
& \leq\|\| \boldsymbol{w}(x-\boldsymbol{t}, \xi)\left(V_{\boldsymbol{\varphi}} \boldsymbol{f}\right)(x-\boldsymbol{t}, \boldsymbol{\xi}) w_{0}(x, \nu+S(\boldsymbol{\xi}))\left(V_{\varphi} g\right)(x, \nu+S(\boldsymbol{\xi}))\left\|_{L^{r_{0}, \boldsymbol{r}}(x, \boldsymbol{t})}\right\|_{L^{\boldsymbol{s}, s_{m+1}(\boldsymbol{\xi}, \nu)}} \\
& \leq\|\| \boldsymbol{w}(\boldsymbol{t}, \xi)\left(V_{\boldsymbol{\varphi}} \boldsymbol{f}\right)(\boldsymbol{t}, \boldsymbol{\xi})\left\|_{L^{\boldsymbol{p}}(\boldsymbol{t})}\right\| w_{0}(x, \nu+S(\boldsymbol{\xi}))\left(V_{\varphi} g\right)(x, \nu+S(\boldsymbol{\xi}))\left\|_{L^{p_{0}}(x)}\right\|_{L^{\boldsymbol{s}, s_{m+1}(\boldsymbol{\xi}, \nu)}} \\
& \leq\|\| \boldsymbol{w}(\boldsymbol{t}, \xi)\left(V_{\boldsymbol{\varphi}} \boldsymbol{f}\right)(\boldsymbol{t}, \boldsymbol{\xi})\left\|_{L^{\boldsymbol{p}}(\boldsymbol{t})}\right\|_{L^{\boldsymbol{q}}(\boldsymbol{\xi})} \\
& \quad\|\| w_{0}(x, \nu+S(\boldsymbol{\xi}))\left(V_{\varphi} g\right)(x, \nu+S(\boldsymbol{\xi}))\left\|_{L^{p_{0}(x)}}\right\|_{L^{q_{m+1}(\nu)}} \\
& =\left\|V_{\boldsymbol{\varphi}} \boldsymbol{f}\right\|_{L_{\boldsymbol{w}}^{\boldsymbol{p}, \boldsymbol{q}}}\left\|V_{\varphi} g\right\|_{L_{w_{0}}^{p_{0} q_{m+1}}} .
\end{aligned}
$$


We now use that $p \leq \widetilde{p}$ and $q \leq \widetilde{q}$ implies $\|f\|_{M^{\tilde{p}, \widetilde{q}}} \lesssim\|f\|_{M^{p, q}}$, a property that clearly carries through to the class of weighted modulation spaces considered in this paper. If hypotheses (2) and (4) hold with strict inequalities, then we can increase $p_{0}$ to appropriate $\widetilde{p}_{0}$ and $q_{m+1}$ to appropriate $\widetilde{q}_{m+1}$ so that (2) and (4) will hold with equalities. The resulting inequalities involving $\widetilde{p}_{0}$ and $\widetilde{q}_{m+1}$ then again implies the weaker inequalities involving $p_{0}$ and $q_{m+1}$.

Example 3.2. The conditions $r_{0} \leq p_{k} \leq r_{k}, k=1, \ldots, m$, and $\sum_{\ell=1}^{m} 1 / p_{\ell}-1 / r_{\ell} \geq 1 / r_{0}-1 / p_{0}$ do not guarantee the existence of a permutation $\kappa$ so that also $\sum_{\ell=1}^{k} 1 / p_{\kappa(\ell)}-1 / r_{\kappa(\ell)} \geq 1 / r_{0}-$ $1 / p_{\kappa(k+1)}, k=0, \ldots, m-1$. Indeed, consider for $m=2$ the case $r_{0}=1, p_{1}=p_{2}=10 / 9$, $r_{1}=r_{2}=2$, and $p_{0}=5$. It is easy to see that no $\kappa$ exist that allows us to apply Proposition 3.1 to obtain for these parameters (3.2). Using $r_{0}=1, p_{1}=p_{2}=10 / 9, r_{1}=r_{2}=2, r_{1}=r_{2}=2$, we can choose $\kappa(0)=1, \kappa(1)=0, \kappa(2)=2$, to obtain (3.2) for $p_{0} \leq 5 / 3$.

Unfortunately, this is again not the best we can do. In fact, we can replace $p_{2}$ by $\widetilde{p}_{2}=$ $15 / 9 \in[10 / 9,18 / 9]=\left[p_{2}, r_{2}\right]$. This choice allows us to choose for $\kappa$ the identity which leads to sufficiency for $p_{0} \leq 2$, which by inclusion also gives boundedness with $r_{0}=1, p_{1}=p_{2}=10 / 9$, $r_{1}=r_{2}=2, r_{1}=r_{2}=2$.

Remark 3.3. Observe those $k$ with $r_{0}>r_{k}$ must satisfy $\kappa^{-1}(k)<z$; possibly there are also $k$ with $r_{0} \leq r_{k}$ and $\kappa^{-1}(k)<z$. Importantly, only those $k$ with $p_{k}<r_{k}$ and $\kappa^{-1}(k)>z$ contribute to filling the gap between $p_{0}$ and $r_{0}$, see Remark 2.3

As immediate consequence, we obtain our first main result.

Theorem 3.4. Given $1 \leq p_{0}, \boldsymbol{p}, \widetilde{\boldsymbol{p}}, \boldsymbol{q}, \widetilde{\boldsymbol{q}}, q_{m+1}, r_{0}, \boldsymbol{r}, \boldsymbol{s}, s_{m+1} \leq \infty$ with $\boldsymbol{p} \leq \widetilde{\boldsymbol{p}} \leq \boldsymbol{r}^{\prime}$ and $\boldsymbol{q}, \boldsymbol{s}^{\prime} \leq \widetilde{\boldsymbol{q}}$. Let $\kappa$ be a permutation on $\{0, \ldots, m\}$ and let $z=\kappa^{-1}(0)$. Similarly, let $\rho$ be a permutation on $\{1,2, \ldots, m+1\}$ and $w=\rho^{-1}(m+1)$ and
(1) $\frac{1}{r_{0}}+\frac{1}{\widetilde{p}_{\kappa(k+1)}}+\sum_{\ell=z+1}^{k} \frac{1}{\widetilde{p}_{\kappa(\ell)}}+\frac{1}{r_{\kappa(\ell)}} \leq k-z+1, k=z, \ldots, m-1 ;$
(2) $\frac{1}{r_{0}}+\sum_{\ell=z+1}^{m} \frac{1}{\widetilde{p}_{\kappa(\ell)}}+\frac{1}{r_{\kappa(\ell)}} \geq m-z+\frac{1}{p_{0}}$;
(3) $\frac{1}{s_{m+1}}+\frac{1}{\widetilde{q}_{\rho(k)}}+\sum_{\ell=k+1}^{w-1} \frac{1}{\widetilde{q}_{\rho(\ell)}}+\frac{1}{s_{\rho(\ell)}} \geq w-k, \quad k=1, \ldots, w-1$;
(4) $\frac{1}{s_{m+1}}+\sum_{\ell=1}^{w-1} \frac{1}{\widetilde{q}_{\rho(\ell)}}+\frac{1}{s_{\rho(\ell)}} \geq w-1+\frac{1}{q_{m+1}}$.

Let $v$ be a weight function on $\mathbb{R}^{2(m+1) d}$ and assume that $w_{0}, w_{1}, \ldots, w_{m}$ are weights on $\mathbb{R}^{2 d}$ such that

$$
v(x, \boldsymbol{t},-\boldsymbol{\xi}, \nu)^{-1} \leq w_{0}(x, \nu+S(\xi))^{-1} w_{1}\left(x-t_{1}, \xi_{1}\right) \cdot \ldots \cdot w_{m}\left(x-t_{m}, \xi_{m}\right) .
$$

Assume that $\sigma \in \mathcal{M}_{v}^{\left(r_{0}, \mathbf{r}\right), \kappa ;\left(\mathbf{s}, s_{m+1}\right), \rho}$. Then the multilinear pseudodifferential operator $T_{\sigma}$ defined initially for $f_{k} \in S\left(\mathbb{R}^{d}\right)$ for $k=1,2, \ldots, m$ by (1.2) extends to a bounded multilinear operator from

$$
M_{w_{1}}^{p_{1}, q_{1}} \times M_{w_{2}}^{p_{2}, q_{2}} \times \ldots \times M_{w_{m}}^{p_{m}, q_{m}} \quad \text { into } \quad M_{w_{0}}^{p_{0}, q_{m+1}} .
$$

Moreover, there exists a constant $C$ so that for all $\boldsymbol{f}$, we have

$$
\left\|T_{\sigma} \boldsymbol{f}\right\|_{M_{w_{0}}^{p_{0} q_{m+1}}} \leq C\|\sigma\|_{\mathcal{M}_{v}^{\left(r_{0}, \mathbf{r}\right), \kappa ;\left(\mathbf{s}, s_{m+1}\right), \rho}}\left\|f_{1}\right\|_{M_{w_{1}}^{p_{1}, q_{1}} \ldots \| f_{m}} \|_{M_{w_{m}}^{p_{m}, q_{m}}}
$$


Proof. Let $f_{k} \in M_{w_{k}}^{p_{k}, q_{k}}, k=1, \ldots m, \varphi \in S\left(\mathbb{R}^{d}\right)$, and denote $\boldsymbol{\varphi}=(\varphi, \ldots, \varphi)$. Note that

$$
\sup \left\{|\langle\cdot, g\rangle|, \quad g \in M_{1 / w_{0}}^{p_{0}^{\prime}, q_{m+1}^{\prime}}\right\}
$$

defines a norm which is equivalent to $\|\cdot\|_{M_{w_{0}}^{p_{0} q_{m+1}}}$ for $p_{0}, q_{m+1} \in[1, \infty]$ (see, for example, 65, Proposition 1.2(3)]). Hence, to complete our result on the basis of Lemma 2.1, we estimate for $g \in M_{1 / w_{0}}^{p_{0}^{\prime}, q_{m+1}^{\prime}}$ as follows

$$
\begin{aligned}
\left|\left\langle T_{\sigma} \boldsymbol{f}, g\right\rangle\right| & =|\langle\sigma, \overline{R(\boldsymbol{f}, g)}\rangle|=\mid\left\langle V_{\overline{R(\boldsymbol{\varphi}, \varphi)}} \sigma, V_{\overline{R(\boldsymbol{\varphi}, \varphi)}} \overline{R(\boldsymbol{f}, g)}\right\rangle \\
& \leq\|\sigma\|_{\mathcal{M}_{v}^{\left(r_{0}, \mathbf{r}\right), \kappa ;\left(\mathbf{s}, s_{m+1}\right), \rho}}\|R(\boldsymbol{f}, g)\|_{\mathcal{M}_{1 / w}^{\left(r_{0}^{\prime}, \boldsymbol{r}^{\prime}\right)_{\kappa},\left(\boldsymbol{s}^{\prime}, s_{m+1}^{\prime}\right)_{\rho}}} .
\end{aligned}
$$

Using the conjugate indices $r_{0}^{\prime}, \mathbf{r}_{\kappa}^{\prime}, s_{m+1}^{\prime}, \mathbf{s}_{\rho}^{\prime}$, it is easy to see that the conditions on the indices (1)-(4) are equivalent to those in Proposition 3.1. Therefore,

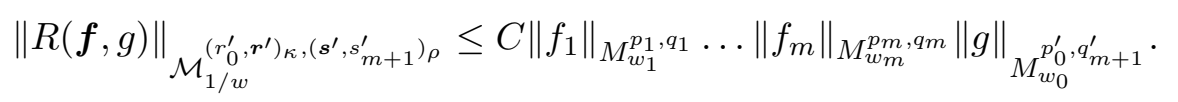

Note that the criteria on time and frequency are separated. Even when it comes to order of integration, we do not link these, that is, the permutations $\kappa$ and $\rho$ are not necessarily identical.

Corollary 3.5. If

$$
\begin{aligned}
& 1 \leq r_{0}^{\prime} \leq p_{1} \leq r_{1}^{\prime} \leq p_{2} \leq \ldots \leq r_{m-1}^{\prime} \leq p_{m} \leq r_{m}^{\prime} \leq \infty ; \\
& 1 \leq s_{1}^{\prime} \leq q_{1} \leq s_{2}^{\prime} \leq q_{2} \leq \ldots \leq q_{m-1} \leq s_{m}^{\prime} \leq q_{m} \leq s_{m+1}^{\prime} \leq \infty
\end{aligned}
$$

and

$$
\begin{gathered}
\frac{1}{r_{0}}+\sum_{\ell=1}^{m} \frac{1}{p_{\ell}}+\frac{1}{r_{\ell}} \geq m+\frac{1}{p_{0}} ; \\
\frac{1}{s_{m+1}}+\sum_{\ell=1}^{m} \frac{1}{q_{\ell}}+\frac{1}{s_{\ell}} \geq m+\frac{1}{q_{m+1}} ;
\end{gathered}
$$

then the conclusion of Theorem 3.4 for any symbol $\sigma \in \mathcal{M}_{v}^{\left(r_{0}, \boldsymbol{r}\right)_{\kappa},\left(\boldsymbol{s}, s_{0}\right)_{\rho}}$, where $\kappa, \rho$ are the identity permutations.

Proof. Note that since $\kappa, \rho$ are the identity permutations, then $z=0$ and $\omega=m+1$.

(1) $\frac{1}{r_{0}}+\frac{1}{p_{k+1}}+\sum_{\ell=1}^{k} \frac{1}{p_{\ell}}+\frac{1}{r_{\ell}} \leq k+1, k=0, \ldots, m-1 ;$
(2) $\frac{1}{s_{m+1}}+\frac{1}{q_{k}}+\sum_{\ell=k+1}^{m} \frac{1}{q_{\ell}}+\frac{1}{s_{\ell}} \geq m-k+1, \quad k=1, \ldots, m$,

follow from the monotonicity conditions.

\section{Applications}

In Section 4.1 we simplify the conditions of Theorem 3.4 in case of bilinear operators, that is, $m=2$. The focus of Section 4.2 lies on establishing boundedness of the bilinear Hilbert transform on products of modulation spaces. We stress that these results are beyond the reach of existing methods of time-frequency analysis of bilinear pseudodifferential operators as developed in [7, 6, 8, 9]. Finally, in Section 4.3 we consider the trilinear Hilbert transform. 
4.1. Bilinear pseudodifferential operators. A bilinear pseudodifferential operator with symbol $\sigma$ is formally defined by

$$
T_{\sigma}(f, g)(x)=\iint_{\mathbb{R} \times \mathbb{R}} \sigma\left(x, \xi_{1}, \xi_{2}\right) \hat{f}\left(\xi_{1}\right) \hat{g}\left(\xi_{2}\right) d \xi_{1} d \xi_{2} .
$$

For $m=2$, Theorem 3.4 simplifies to the following.

Theorem 4.1. Let $1 \leq p_{0}, p_{1}, p_{2}, q_{1}, q_{2}, q_{3}, r_{0}, r_{1}, r_{2}, s_{1}, s_{2}, s_{3} \leq \infty$. If

$$
1 / p_{1}+1 / r_{1}, 1 / p_{2}+1 / r_{2} \geq 1
$$

and one of the following

$$
\begin{array}{rlrl}
\frac{1}{p_{0}} & \leq \frac{1}{r_{0}}, & (\text { using } \kappa=(1,2,0) \text { or }(2,1,0)) ; \\
1+\frac{1}{p_{0}} & \leq \frac{1}{r_{0}}+\frac{1}{r_{1}}+\frac{1}{p_{1}}, \quad r_{1} \leq p_{0}, r_{0}, \quad(\kappa=(2,0,1)) ; \\
1+\frac{1}{p_{0}} & \leq \frac{1}{r_{0}}+\frac{1}{r_{2}}+\frac{1}{p_{2}}, \quad r_{2} \leq p_{0}, r_{0}, \quad(\kappa=(1,0,2)) ; \\
2+\frac{1}{p_{0}} & \leq \frac{1}{r_{0}}+\frac{1}{r_{1}}+\frac{1}{r_{2}}+\frac{1}{\max \left\{p_{1}, r_{0}^{\prime}\right\}}+\frac{1}{p_{2}}, \quad r_{2} \leq p_{0}, \quad r_{1}, r_{2} \leq r_{0}, \quad(\kappa=(0,1,2)) ; \\
2+\frac{1}{p_{0}} & \leq \frac{1}{r_{0}}+\frac{1}{r_{1}}+\frac{1}{r_{2}}+\frac{1}{\max \left\{p_{2}, r_{0}^{\prime}\right\}}+\frac{1}{p_{1}}, \quad r_{1} \leq p_{0}, \quad r_{1}, r_{2} \leq r_{0}, \quad(\kappa=(0,2,1)) ;
\end{array}
$$

as well as one of

$$
\begin{aligned}
\frac{1}{q_{3}} & \leq \frac{1}{s_{3}}, \quad(\text { using } \rho=(3,1,2) \text { or }(3,2,1)) \\
1+\frac{1}{q_{3}} & \leq \frac{1}{q_{1}}+\frac{1}{s_{1}}+\frac{1}{s_{3}}, \quad s_{3} \leq q_{3}, s_{1}, q_{1}^{\prime}, \quad(\rho=(1,3,2)) ; \\
1+\frac{1}{q_{3}} & \leq \frac{1}{q_{2}}+\frac{1}{s_{2}}+\frac{1}{s_{3}}, \quad s_{3} \leq q_{3}, s_{2}, q_{2}^{\prime}, \quad(\rho=(2,3,1)) ; \\
2 & \leq \frac{1}{\max \left\{q_{1}, s_{1}^{\prime}\right\}}+\frac{1}{\max \left\{q_{2}, s_{2}^{\prime}\right\}}+\frac{1}{s_{2}}+\frac{1}{s_{3}}, \quad s_{3} \leq q_{2}^{\prime}, s_{2}, \\
2+\frac{1}{q_{3}} & \leq \frac{1}{\max \left\{q_{1}, s_{1}^{\prime}\right\}}+\frac{1}{\max \left\{q_{2}, s_{2}^{\prime}\right\}}+\frac{1}{s_{1}}+\frac{1}{s_{2}}+\frac{1}{s_{3}}, \quad(\rho=(1,2,3)) ; \\
2 & \leq \frac{1}{\max \left\{q_{1}, s_{1}^{\prime}\right\}}+\frac{1}{\max \left\{q_{2}, s_{2}^{\prime}\right\}}+\frac{1}{s_{1}}+\frac{1}{s_{3}}, \quad s_{3} \leq q_{1}^{\prime}, s_{1}, \\
2+\frac{1}{q_{3}} & \leq \frac{1}{\max \left\{q_{1}, s_{1}^{\prime}\right\}}+\frac{1}{\max \left\{q_{2}, s_{2}^{\prime}\right\}}+\frac{1}{s_{1}}+\frac{1}{s_{2}}+\frac{1}{s_{3}}, \quad(\rho=(1,3,2)),
\end{aligned}
$$

hold. Assume that $w_{0}, w_{1}, w_{2}$, and $v$ are weight functions satisfying

$$
v\left(x, t_{1}, t_{2}, \xi_{1}, \xi_{2}, \nu\right)^{-1} \leq w_{0}\left(x, \nu+\xi_{1}+\xi_{2}\right)^{-1} \cdot w_{1}\left(x-t_{1}, \xi_{1}\right) \cdot w_{2}\left(x-t_{2}, \xi_{2}\right) .
$$

If $\sigma \in \mathcal{M}^{\left(r_{0}, r_{1}, r_{2}\right), \kappa ;\left(s_{1}, s_{2}, s_{3}\right), \rho}$, the bilinear pseudodifferential operator $T_{\sigma}$ initially defined on $S\left(\mathbb{R}^{d}\right) \times S\left(\mathbb{R}^{d}\right)$ by (4.1) extends to a bounded bilinear operator from $M_{w_{1}}^{p_{1}, q_{1}} \times M_{w_{2}}^{p_{2}, q_{2}}$ into $M_{w_{0}}^{p_{0}, q_{3}}$. Moreover, there exists a constant $C>0$, such that we have

$$
\left\|T_{\sigma}\left(f_{1}, f_{2}\right)\right\|_{M_{w_{0}}^{p_{0}, q_{3}}} \leq C\|\sigma\|_{\mathcal{M}^{\left(r_{0}, r_{1}, r_{2}\right), \kappa ;\left(s_{1}, s_{2}, s_{3}\right), \rho}}\left\|f_{1}\right\|_{M_{w_{1}}^{p_{1}, q_{1}}}\left\|f_{2}\right\|_{M_{w_{2}}^{p_{2}, q_{2}}}
$$

with appropriately chosen order of integration $\kappa, \rho$. 
Proof. This result is derived from Theorem 3.4 for $m=2$, namely, we establish conditions on the $p_{0}, p_{1}, p_{2}, r_{0}, r_{1}, r_{2}, q_{1}, q_{2}, q_{3}, s_{1}, s_{2}, s_{3}$ for the existence of $\widetilde{p}_{1}, \widetilde{p}_{2}, \widetilde{q}_{1}, \widetilde{q}_{2}$ satisfying the conditions of Theorem 3.4 .

If $\kappa=\left(\begin{array}{lll}1 & 2 & 0\end{array}\right)$ or $\kappa=\left(\begin{array}{lll}2 & 1 & 0\end{array}\right)$, then $z=2$ in Theorem 3.4 and we require in addition only $\frac{1}{r_{0}} \geq \frac{1}{p_{0}}$.

For the remaining cases, we have to show that the conditions above imply the existence of $\widetilde{p}_{1} \geq p_{1}, \widetilde{p}_{2} \geq p_{2}$ which allow for the application of Theorem 3.4 .

If $\kappa=\left(\begin{array}{lll}1 & 0 & 2\end{array}\right)$, we have $z=1$, and we seek, with notation as before, $\widetilde{P}_{1}$ and $\widetilde{P}_{2}$ with

$$
\begin{aligned}
\widetilde{P}_{1} & \leq P_{1} ; & \widetilde{P}_{2} \leq P_{2} ; \\
\widetilde{P}_{1}+R_{1} & \geq 1 ; & \widetilde{P}_{2}+R_{2} \geq 1 ; \\
R_{0}+\widetilde{P}_{2} & \leq 1 ; & \\
R_{0}+\widetilde{P}_{2}+R_{2} & \geq 1+P_{0} ; &
\end{aligned}
$$

that is,

$$
\begin{gathered}
1-R_{1} \leq \widetilde{P}_{1} \leq P_{1} \\
1-R_{0}-R_{2}+P_{0}, 1-R_{2} \leq \widetilde{P}_{2} \leq P_{2}, 1-R_{0}
\end{gathered}
$$

which defines a non empty set if and only if $P_{1}+R_{1} \geq 1, P_{2}+R_{2} \geq 1, R_{2} \geq P_{0}, R_{0}, 1+P_{0} \leq$ $R_{0}+R_{1}+P_{2}$.

For $\kappa=\left(\begin{array}{lll}0 & 1 & 2\end{array}\right)$ we have $z=0$ in Theorem 3.4 and we require that some $\widetilde{P}_{1}$ and $\widetilde{P}_{2}$ satisfy

$$
\begin{array}{rr}
\widetilde{P}_{1} \leq P_{1} ; & \widetilde{P}_{2} \leq P_{2} ; \\
\widetilde{P}_{1}+R_{1} \geq 1 ; & \widetilde{P}_{2}+R_{2} \geq 1 ; \\
R_{0}+\widetilde{P}_{2} \leq 1 ; & R_{0}+\widetilde{P}_{2}+\widetilde{P}_{1}+R_{1} \leq 2 ; \\
R_{0}+\widetilde{P}_{2}+R_{2}+\widetilde{P}_{1}+R_{1} \geq 2+P_{0} ; &
\end{array}
$$

that is,

$$
\begin{aligned}
1-R_{1} & \leq \widetilde{P}_{1} \leq P_{1}, 1-R_{0} ; \\
1-R_{2} & \leq \widetilde{P}_{2} \leq P_{2} ; \\
2+P_{0}-R_{0}-R_{1}-R_{2} & \leq \widetilde{P}_{1}+\widetilde{P}_{2} \leq 2-R_{0}-R_{1} .
\end{aligned}
$$

Note that (4.3) defines a vertical strip in the $\left(\widetilde{P}_{1}, \widetilde{P}_{2}\right)$ plane which is non-empty if and only if $P_{1}+R_{1} \geq 1$ and $R_{0} \leq R_{1}$. Similarly, (4.4) defines a horizontal strip which is not empty if we assume $P_{2}+R_{2} \geq 1$. Lastly, the diagonal strip given by (4.5) is nonempty if and only if $P_{0} \leq R_{2}$.

To obtain a boundedness result, we still need to establish that the diagonal strip meats the rectangle given by the intersection of horizontal and vertical strips. This is the case if the upper right hand corner of the rectangle is above the lower diagonal given by $\widetilde{P}_{1}+\widetilde{P}_{2}=$ $2+P_{0}-R_{0}-R_{1}-R_{2}$, that is, if

$$
\min \left\{P_{1}, 1-R_{0}\right\}+P_{2} \geq 2+P_{0}-R_{0}-R_{1}-R_{2},
$$

and if the lower left corner of the rectangle lies below the upper diagonal, that is, if

$$
1-R_{1}+1-R_{2} \leq 2-R_{0}-R_{1},
$$


which holds if $R_{0} \leq R_{2}$.

Let us now turn to the frequency side. If $\rho=\left(\begin{array}{lll}3 & 2 & 1\end{array}\right)$ or $\rho=\left(\begin{array}{lll}3 & 1 & 2\end{array}\right)$, we have $w=1$ and an application Theorem 3.4 requires the single but strong assumption $Q_{3} \leq S_{3}$.

For $\rho=\left(\begin{array}{lll}1 & 3 & 2\end{array}\right)$ we have $w=2$ in Theorem 3.4. To satifiy the conditions, we need to establish the existence of $\widetilde{Q}_{1}$ and $\widetilde{Q}_{2}$ satisfy

$$
\begin{array}{rlrl}
\widetilde{Q}_{1} & \leq Q_{1} ; & \widetilde{Q}_{2} \leq Q_{2} ; \\
\widetilde{Q}_{1}+S_{1} \leq 1 ; & \widetilde{Q}_{2}+S_{2} \leq 1 ; \\
S_{3}+\widetilde{Q}_{1} \geq 1 ; & S_{3}+\widetilde{Q}_{1}+S_{1} \geq 1+Q_{3} .
\end{array}
$$

The existence of such $\widetilde{Q}_{2}$ is trivial, so we are left with

$$
1+Q_{3}-S_{1}-S_{3}, 1-S_{3} \leq \widetilde{Q}_{1} \leq Q_{1}, 1-S_{1}, .
$$

Note that this inequality is exactly (4.2) with $S_{3}$ replacing $R_{2}, S_{1}$ replacing $R_{0} Q_{3}$ replacing $P_{0}$, and $Q_{1}, \widetilde{Q}_{1}$ in place of $P_{2}, \widetilde{P}_{2}$.

We conclude that for the existence of $\widetilde{Q}_{1}$, we require $S_{3} \geq S_{1}, Q_{3}, 1-Q_{1}$, and $1+Q_{3} \leq$ $Q_{1}+S_{1}+S_{3}$.

For $\rho=\left(\begin{array}{lll}1 & 2 & 3\end{array}\right)$ we have $w=3$ in Theorem 3.4. We need to establish the existence of $\widetilde{Q}_{1}$ and $\widetilde{Q}_{2}$ satisfy

$$
\begin{array}{rlrl}
\widetilde{Q}_{1} & \leq Q_{1} ; & \widetilde{Q}_{2} \leq Q_{2} ; \\
\widetilde{Q}_{1}+S_{1} & \leq 1 ; \\
S_{3}+\widetilde{Q}_{2} \geq 1 ; & \widetilde{Q}_{2}+S_{2} \leq 1 ; \\
S_{3}+\widetilde{Q}_{1}+\widetilde{Q}_{2}+S_{2}+S_{1} \geq 2+Q_{3} ; & S_{3}+\widetilde{Q}_{2}+\widetilde{Q}_{1}+S_{2} \geq 2 ;
\end{array}
$$

that is, choosing

$$
\widetilde{Q}_{1}=\min \left\{Q_{1}, 1-S_{1}\right\}, \quad \text { and } \quad \widetilde{Q}_{2}=\min \left\{Q_{2}, 1-S_{2}\right\}
$$

we require

$$
\begin{aligned}
1 & \leq \min \left\{Q_{2}, 1-S_{2}\right\}+S_{3} ; \\
2 & \leq \min \left\{Q_{1}, 1-S_{1}\right\}+\min \left\{Q_{2}, 1-S_{2}\right\}+S_{2}+S_{3} ; \\
2+Q_{3} & \leq \min \left\{Q_{1}, 1-S_{1}\right\}+\min \left\{Q_{2}, 1-S_{2}\right\}+S_{1}+S_{2}+S_{3} .
\end{aligned}
$$

Proof. Proof of Theoremm 1.1 Theorem 1.1 now follows from choosing $\kappa$ and $\rho$ to be the identity permutations, and $r_{0}=s_{1}=s_{2}=\infty, r_{1}=r_{2}=s_{3}=1$.

Note that this result covers and extends Theorem 3.1 in [7].

Remark 4.2. Using Remark 2.8, we observe that $M^{\infty, 1}\left(\mathbb{R}^{3 d}\right) \subsetneq \mathcal{M}^{(\infty, 1,1),(\infty, \infty, 1)}\left(\mathbb{R}^{3 d}\right)$. Indeed, in both cases we have the same decay parameters, but different integration orders, namely

$$
\begin{array}{lllll}
M^{\infty, 1} & x \rightarrow \infty, \quad \xi_{1} \rightarrow \infty, & \xi_{2} \rightarrow \infty, & \nu \rightarrow 1, \quad t_{1} \rightarrow 1, \quad t_{2} \rightarrow 1 \\
\mathcal{M}^{(\infty, 1,1),(\infty, \infty, 1)} & x \rightarrow \infty, \quad t_{1} \rightarrow 1, & t_{2} \rightarrow 1, & \xi_{1} \rightarrow \infty, & \xi_{2} \rightarrow \infty, \quad \nu \rightarrow 1 .
\end{array}
$$

Inclusion follows from the fact that we always moved a large exponent to the right of a small exponent. Note that for any $r \in M^{1, \infty}(\mathbb{R}) \backslash M^{\infty, 1}(\mathbb{R})$, for example, a chirped signal $r(\xi)=$ $e^{2 \pi i \xi^{2}} u(\xi)$ with $u(\xi) \in L^{2} \backslash L^{1}$, we have

$$
\sigma\left(x, \xi_{1}, \xi_{2}\right)=r\left(\xi_{1}\right) \in \mathcal{M}^{(\infty, 1,1),(\infty, \infty, 1)} \backslash M^{\infty, 1} .
$$


Example 4.3. With $\kappa$ and $\rho$ are the identity, that is, $\kappa=(0,1,2)$ and $\rho=(1,2,3)$, we illustrate the applicability of Theorem 4.1 for maps on $L^{2} \times L^{2}=M^{2,2} \times M^{2,2}$, that is, $p_{1}=p_{2}=q_{1}=$ $q_{2}=2$.

On the time side, we require $r_{1}, r_{2} \leq 2, r_{0}$ and $r_{2} \leq p_{0}$ as well as

$$
\frac{3}{2}+\frac{1}{p_{0}} \leq \frac{1}{r_{0}}+\frac{1}{r_{1}}+\frac{1}{r_{2}}+\frac{1}{\max \left\{2, r_{0}^{\prime}\right\}} .
$$

Our goal is to obtain results for $r_{0}$ large, hence, we assume $r_{0} \geq 2$. (In case of $r_{0} \leq 2$, the last inequality above does not depend on $r_{0}$, and we can improve the result by fixing $r_{0}=2$.) We obtain the range of applicability $r_{1}, r_{2} \leq 2 \leq r_{0}$, and $r_{2} \leq p_{0}$, and

$$
1+\frac{1}{p_{0}} \leq \frac{1}{r_{0}}+\frac{1}{r_{1}}+\frac{1}{r_{2}}
$$

On the frequency side, we have to satisfy the conditions $s_{3} \leq 2, s_{2}$,

$$
\begin{gathered}
2 \leq \frac{1}{\max \left\{2, s_{1}^{\prime}\right\}}+\frac{1}{\max \left\{2, s_{2}^{\prime}\right\}}+\frac{1}{s_{2}}+\frac{1}{s_{3}}, \\
2+\frac{1}{q_{3}} \leq \frac{1}{\max \left\{2, s_{1}^{\prime}\right\}}+\frac{1}{\max \left\{2, s_{2}^{\prime}\right\}}+\frac{1}{s_{1}}+\frac{1}{s_{2}}+\frac{1}{s_{3}} .
\end{gathered}
$$

Let us assume $s_{1} \leq 2 \leq s_{2}$, then we have the range of applicability $s_{1}, s_{3} \leq 2 \leq s_{2}$,

$$
\frac{1}{2}+\frac{1}{s_{1}}, \frac{1}{2}+\frac{1}{q_{3}} \leq \frac{1}{s_{2}}+\frac{1}{s_{3}} .
$$

The range of applicability gives exponents that guarantee that a bilinear pseudodifferential operator maps boundedly $L^{2} \times L^{2}$ into $M^{p_{0}, q_{3}}$ if $\sigma \in \mathcal{M}^{\left(r_{0}, r_{1}, r_{2}\right),\left(s_{1}, s_{2}, s_{3}\right)}$.

In particular, when $\sigma \in \mathcal{M}^{(\infty, 1,1),(2,2,1)}$ we can take $p_{0}=q_{3}=1$. So we get that $T_{\sigma}$ maps $L^{2} \times L^{2}$ into $M^{1,1} \subset M^{1, \infty}$.

4.2. The bilinear Hilbert transform. We now consider boundedness properties of the bilinear Hilbert transform on modulation spaces. Recall that this operator is defined for $f, g \in \mathcal{S}(\mathbb{R})$ by

$$
\mathrm{BH}(f, g)(x)=\lim _{\epsilon \rightarrow 0} \int_{|y|>\epsilon} f(x+y) g(x-y) \frac{d y}{y} .
$$

Equivalently, this operator can be written as a Fourier multiplier, that is, a bilinear pseudodifferential operator whose symbol is independent of the space variable, with symbol $\sigma_{\mathrm{BH}}\left(x, \xi_{1}, \xi_{2}\right)=$ $\sigma\left(\xi_{1}-\xi_{2}\right)$, where $\sigma(x)=-\pi i \operatorname{sign}(x), x \neq 0$.

Our first goal is to identify which of the (unweighted) spaces $\mathcal{M}^{\left(r_{0}, r_{1}, r_{2}\right), \kappa ;\left(s_{1}, s_{2}, s_{0}\right), \rho}$ the symbol $\sigma_{\mathrm{BH}}$ belongs to. To this end consider the window function $\Psi\left(x, \xi_{1}, \xi_{2}\right)=\psi(x) \psi\left(\xi_{2}\right) \psi\left(\xi_{1}-\xi_{2}\right)$, where $\psi \in \mathcal{S}(\mathbb{R})$ such that $\psi(x)=\psi_{1}(x)-\psi_{1}(-x)$ with $\psi_{1} \in \mathcal{S}(\mathbb{R}), 0 \leq \psi_{1}(x) \leq 1$ for all $x \in \mathbb{R}$. In addition, we require that the support of $\psi_{1}$ is strictly included in $(0,1)$. Then

$$
\mathcal{V}_{\Psi} \sigma_{\mathrm{BH}}\left(x, t_{1}, t_{2}, \xi_{1}, \xi_{2}, \nu\right)=V_{\psi} 1(x, \nu) V_{\psi} \sigma\left(\xi_{1}-\xi_{2}, t_{1}\right) V_{\psi} 1\left(\xi_{2}, t_{1}+t_{2}\right)
$$

Assume that the two permutations $\kappa$ of $\{0,1,2\}$, and $\rho$ of $\{1,2,3\}$ are identities. Moreover, suppose that all the weights are identically equal to 1 .

Proposition 4.4. For $r>1$, we have that $\sigma_{B H} \in \mathcal{M}^{(\infty, 1, r),(\infty, \infty, 1)}$.

Proof. Let $r>1$. We shall integrate

$$
\mathcal{V}_{\Psi} \sigma_{\mathrm{BH}}(x, \boldsymbol{t}, \boldsymbol{\xi}, \nu)=V_{\psi} 1(x, \nu) V_{\psi} \sigma\left(\xi_{1}-\xi_{2}, t_{1}\right) V_{\psi} 1\left(\xi_{2}, t_{1}+t_{2}\right)
$$


in the order

$$
x \rightarrow r_{0}=\infty \quad t_{1} \rightarrow r_{1}=1 \quad t_{2} \rightarrow r_{2}=r>1 \quad \xi_{1} \rightarrow s_{1}=\infty \quad \xi_{2} \rightarrow s_{2}=\infty \quad \nu \rightarrow s_{0}=1 .
$$

We estimate

$$
\begin{aligned}
\left\|\sigma_{\mathrm{BH}}\right\|_{\mathcal{M}^{(\infty, \infty, 1),(\infty, 1, r)}} & =\int_{\mathbb{R}} \sup _{\xi_{1}, \xi_{2}}\left(\int_{\mathbb{R}}\left(\int_{\mathbb{R}} \sup _{x}\left|\mathcal{V}_{\Psi} \sigma_{\mathrm{BH}}(x, \boldsymbol{t}, \boldsymbol{\xi}, \nu)\right| d t_{1}\right)^{r} d t_{2}\right)^{1 / r} d \nu \\
& =\int_{\mathbb{R}} \sup _{\xi_{1}, \xi_{2}}\left(\int_{\mathbb{R}}\left(\int_{\mathbb{R}} \sup _{x}\left|V_{\psi} 1(x, \nu) V_{\psi} \sigma\left(\xi_{1}-\xi_{2}, t_{1}\right) V_{\psi} 1\left(\xi_{2}, t_{1}+t_{2}\right)\right| d t_{1}\right)^{r} d t_{2}\right)^{1 / r} d \nu \\
& =\|\hat{\psi}\|_{L^{1}} \sup _{\xi_{1}, \xi_{2}}\left(\int_{\mathbb{R}}\left(\int_{\mathbb{R}}\left|V_{\psi} \sigma\left(\xi_{1}-\xi_{2}, t_{1}\right) V_{\psi} 1\left(\xi_{2}, t_{1}+t_{2}\right)\right| d t_{1}\right)^{r} d t_{2}\right)^{1 / r} \\
& \leq\|\hat{\psi}\|_{L^{1}} \sup _{\xi_{1}, \xi_{2}}\left\|V_{\psi} \sigma\left(\xi_{1}-\xi_{2}, \cdot\right)|*| V_{\psi} 1\left(\xi_{2}, \cdot\right) \mid\right\|_{L^{r}} \\
& \leq\|\hat{\psi}\|_{L^{1}} \sup _{\xi_{1}, \xi_{2}}\left\|V_{\psi} \sigma\left(\xi_{1}-\xi_{2}, \cdot\right)\right\|_{L^{r}}\left\|V_{\psi} 1\left(\xi_{2}, \cdot\right)\right\|_{L^{1}} \\
& =\|\hat{\psi}\|_{L^{1}}^{2} \sup _{\xi_{1}}\left\|V_{\psi} \sigma\left(\xi_{1} \cdot\right)\right\|_{L^{r}},
\end{aligned}
$$

where we have repeatedly used the fact that $V_{\psi} 1(x, \nu)=e^{2 \pi i x \nu} \hat{\psi}(\nu)$, and $V_{\psi} 1 \in L^{\infty}(x) L^{1}(\nu)$, that is

Thus, we are left to estimate

$$
\int_{\mathbb{R}} \sup _{x}\left|V_{\psi} 1(x, \nu)\right| d \nu=\|\hat{\psi}\|_{L^{1}}<\infty
$$

$$
\sup _{\xi}\left\|V_{\psi} \sigma(\xi \cdot)\right\|_{L^{r}}
$$

Recall that $\psi(x)=\psi_{1}(x)-\psi_{1}(-x)$, hence, we have

$$
V_{\psi} \sigma(\xi, t)=e^{-2 \pi i \xi t}\left[-\int_{-\infty}^{-\xi} e^{-2 \pi i y t} \psi(y) d y+\int_{-\xi}^{\infty} e^{-2 \pi i t y} \psi(y) d y\right]
$$

A series of straightforward calculations yields

$$
\left|V_{\psi} \sigma(\xi, t)\right|= \begin{cases}\left|\hat{\psi}_{1}(t)-\hat{\psi}_{1}(-t)\right| & \text { if }|\xi| \geq 1 \\ \left|\hat{\psi}_{1}(-t)-\widehat{\chi_{[0,-\xi]}} * \hat{\psi}_{1}(t)+\widehat{\chi_{[-\xi, 1]}} * \hat{\psi}_{1}(t)\right| & \text { if }-1 \leq \xi \leq 0 \\ \left|\hat{\psi}_{1}(t)-\widehat{\chi_{[\xi, 1]}} * \hat{\psi}_{1}(-t)+\widehat{\chi_{[0, \xi]}} * \hat{\psi}_{1}(-t)\right| & \text { if } 0 \leq \xi \leq 1\end{cases}
$$

where $\chi_{[a, b]}$ denotes the characteristic function of $[a, b]$. We note that that $\widehat{\chi_{[0,-\xi]}}, \widehat{\chi_{[-\xi, 1]}}, \widehat{\chi_{[\xi, 1]}}, \widehat{\chi_{[0, \xi]}} \in$ $L^{r}$ uniformly for $|\xi| \leq 1$ for each $r>1$.

For $|\xi| \geq 1$, we have

$$
\left\|V_{\psi} \sigma(\xi, \cdot)\right\|_{L^{q}} \leq 2\left\|\hat{\psi}_{1}\right\|_{L^{q}}
$$

for any $q \geq 1$. Now consider $-1 \leq \xi \leq 0$, then

$$
\begin{aligned}
\left\|V_{\psi} \sigma(\xi, \cdot)\right\|_{L^{r}} & \leq\left\|\hat{\psi}_{1}\right\|_{L^{r}}+\left\|\widehat{\chi_{[0,-\xi]}} * \hat{\psi_{1}}\right\|_{L^{r}}+\left\|\widehat{\chi_{[-\xi, 1]}} * \hat{\psi}_{1}\right\|_{L^{r}} \\
& \leq\left\|\hat{\psi}_{1}\right\|_{L^{r}}+\left\|\hat{\psi_{1}}\right\|_{L^{1}}\left(\left\|\widehat{\chi_{[0,-\xi]}}\right\|_{L^{r}}+\left\|\widehat{\chi_{[-\xi, 1]}}\right\|_{L^{r}}\right) \\
& \leq\left\|\hat{\psi}_{1}\right\|_{L^{r}}+C\left\|\hat{\psi}_{1}\right\|_{L^{1}}
\end{aligned}
$$

where $C>0$ is a constant that depends only on $r$. Using a similar estimate for $0 \leq \xi \leq 1$, we conclude that

$$
\sup _{\xi}\left\|V_{\psi} \sigma(\xi, \cdot)\right\|_{L^{r}} \leq C<\infty
$$


where $C$ depends only on $\psi_{1}$ and $r$.

Observe that $\sigma_{\mathrm{BH}} \in \mathcal{M}^{(\infty, 1, r),(\infty, \infty, 1)}\left(\mathbb{R}^{3}\right) \backslash \mathcal{M}^{(\infty, 1,1),(\infty, \infty, 1)}\left(\mathbb{R}^{3}\right)$ for all $r>1$. Consequently, to obtain a boundedness result for the bilinear Hilbert transform, we cannot apply any of the existing results on bilinear pseudodifferential operators. However, using the symbol classes introduced we obtain the following result:

Theorem 4.5. Let $1 \leq p_{0}, p_{1}, p_{2}, q_{1}, q_{2}, q_{3} \leq \infty$ satisfy $\frac{1}{p_{1}}+\frac{1}{p_{2}}>\frac{1}{p_{0}}$ and that $\frac{1}{q_{1}}+\frac{1}{q_{2}} \geq 1+\frac{1}{q_{3}}$. Then the bilinear Hilbert transform extends to a bounded bilinear operator from $M^{p_{1}, q_{1}} \times M^{p_{2}, q_{2}}$ into $M^{p_{0}, q_{3}}$. Moreover, there exists a constant $C>0$ such that

$$
\|B H(f, g)\|_{M^{p_{0}, q_{3}}} \leq C\|f\|_{M^{p_{1}, q_{1}}}\|g\|_{M^{p_{2}, q_{2}}} .
$$

In particular, for any $1 \leq p, q \leq \infty$, and $\epsilon>0$, the $B H$ continuously maps $M^{p, q} \times M^{p^{\prime}, q^{\prime}}$ into $M^{1+\epsilon, \infty}$ and we have

$$
\|B H(f, g)\|_{M^{1+\epsilon, \infty}} \leq C\|f\|_{M^{p, q}}\|g\|_{M^{p^{\prime}, q^{\prime}}} .
$$

Proof. Since the symbol $\sigma_{\mathrm{BH}}$ of $\mathrm{BH}$ satisfies $\sigma_{\mathrm{BH}} \in \mathcal{M}^{(\infty, 1, r),(\infty, \infty, 1)}$, the proof follows from Theorem 4.1. Indeed, on the time side, all simple inequalities hold and we are left to check

$$
2+\frac{1}{p_{0}} \leq \frac{1}{r_{0}}+\frac{1}{r_{1}}+\frac{1}{r_{2}}+\frac{1}{\max \left\{p_{1}, r_{0}^{\prime}\right\}}+\frac{1}{p_{2}}
$$

which is with $\frac{1}{r}=1-\epsilon$

$$
2+\frac{1}{p_{0}} \leq 0+1+1-\epsilon+\frac{1}{\max \left\{p_{1}, 1\right\}}+\frac{1}{p_{2}}
$$

On the frequency side, the conditions

$$
\begin{aligned}
2 & \leq \frac{1}{\max \left\{q_{1}, s_{1}^{\prime}\right\}}+\frac{1}{\max \left\{q_{2}, s_{2}^{\prime}\right\}}+\frac{1}{s_{2}}+\frac{1}{s_{3}}, \quad s_{3} \leq q_{2}^{\prime}, s_{2}, \\
2+\frac{1}{q_{3}} & \leq \frac{1}{\max \left\{q_{1}, s_{1}^{\prime}\right\}}+\frac{1}{\max \left\{q_{2}, s_{2}^{\prime}\right\}}+\frac{1}{s_{1}}+\frac{1}{s_{2}}+\frac{1}{s_{3}},
\end{aligned}
$$

are clearly satisfied whenever

$$
2+\frac{1}{q_{3}} \leq \frac{1}{\max \left\{q_{1}, 1\right\}}+\frac{1}{\max \left\{q_{2}, 1\right\}}+0+0+0 .
$$

Remark 4.6. It was proved in [44, 45] that the bilinear Hilbert transform BH continuously maps $L^{p_{1}} \times L^{p_{2}}$ into $L^{p}$ where $\frac{1}{p}=\frac{1}{p_{1}}+\frac{1}{p_{2}}, 1 \leq p_{1}, p_{2} \leq \infty$ and $2 / 3<p \leq \infty$. Our results give that if $1<p, q, p_{1}<\infty$ then $H$ maps continuously $M^{p_{1}, q} \times M^{p_{1}^{\prime}, q^{\prime}}$ into $M^{p, \infty}$.

One can use embeddings between modulation spaces and Lebesgue spaces to get some "mixed" boundedness results. For example, assume that $q \geq 2$ and $q^{\prime} \leq p_{1} \leq q$, then it is known that (see [60, Proposition 1.7])

$$
L^{p_{1}} \subset M^{p_{1}, q} \quad \text { and } \quad M^{p_{1}^{\prime}, q^{\prime}} \subset L^{p_{1}^{\prime}} .
$$

Consequently, it follows from Theorem 4.5 that BH continuously maps $L^{p_{1}} \times M^{p_{1}^{\prime}, q^{\prime}}$ into $M^{p, \infty} \supset$ $L^{p}$. 
4.3. The trilinear Hilbert transform. In this final section we consider the trilinear Hilbert transform TH given formally by

$$
\mathrm{TH}(f, g, h)(x)=\lim _{\epsilon \rightarrow 0} \int_{|t|>\epsilon} f(x-t) g(x+t) h(x+2 t) \frac{d t}{t} .
$$

The trilinear Hilbert transform can be written as a trilinear pseudodifferential operator, or more specifically as a trilinear Fourier multiplier given by

$$
\mathrm{TH}(f, g, h)(x)=\iiint_{\mathbb{R} \times \mathbb{R} \times \mathbb{R}} \sigma_{\mathrm{TH}}\left(x, \xi_{1}, \xi_{2}, \xi_{3}\right) \hat{f}\left(\xi_{1}\right) \hat{g}\left(\xi_{2}\right) \hat{h}\left(\xi_{3}\right) e^{2 \pi i x\left(\xi_{1}+\xi_{2}+\xi_{3}\right)} d \xi_{1} d \xi_{2} d \xi_{3}
$$

where

$$
\sigma_{\mathrm{TH}}\left(x, \xi_{1}, \xi_{2}, \xi_{3}\right)=\sigma\left(\xi_{1}-\xi_{2}-2 \xi_{3}\right)=\pi i \operatorname{sign}\left(\xi_{1}-\xi_{2}-2 \xi_{3}\right)
$$

Recall from Section 4.2 that $\psi \in \mathcal{S}(\mathbb{R})$ is chosen such that $\psi(x)=\psi_{1}(x)-\psi_{1}(-x)$ with $\psi_{1} \in \mathcal{S}(\mathbb{R}), 0 \leq \psi_{1}(x) \leq 1$ for all $x \in \mathbb{R}$. Next we define $\Psi\left(x, \xi_{1}, \xi_{2}, \xi_{3}\right)=\psi(x) \psi\left(\xi_{2}\right) \psi\left(\xi_{3}\right) \psi\left(\xi_{1}-\right.$ $\left.\xi_{2}-2 \xi_{3}\right)$. We can now compute the symbol window Fourier transform $\mathcal{V}_{\Psi} \sigma_{\mathrm{TH}}$ of $\sigma_{\mathrm{TH}}$ with respect to $\Psi$ and obtain

$$
\mathcal{V}_{\Psi} \sigma_{\mathrm{TH}}(x, \boldsymbol{t}, \boldsymbol{\xi}, \nu)=V_{\psi} 1(x, \nu) V_{\psi} 1\left(\xi_{2},-t_{1}-t_{2}\right) V_{\psi} 1\left(\xi_{2},-2 t_{1}-t_{3}\right) V_{\psi} \sigma\left(\xi_{1}-\xi_{2}-2 \xi_{3},-t_{1}\right) \mid .
$$

Observe that $\left|V_{g} 1(x, \eta)\right|=|\hat{g}(\eta)|$. Hence,

$$
\left|\mathcal{V}_{\Psi} \sigma_{\mathrm{TH}}(x, \boldsymbol{t}, \boldsymbol{\xi}, \nu)\right|=|\hat{\psi}(\nu)|\left|\hat{\psi}\left(-t_{1}-t_{2}\right)\right|\left|\hat{\psi}\left(-2 t_{1}-t_{3}\right)\right|\left|V_{\psi} \sigma\left(\xi_{1}-\xi_{2}-2 \xi_{3},-t_{1}\right)\right| .
$$

But by the choice of $\psi$ we see that $\hat{\psi}(-\eta)=-\hat{\psi}(\eta)$.

Proposition 4.7. For $r>1$, we have $\sigma_{T H} \in \mathcal{M}^{(\infty, 1, r, r),(\infty, \infty, \infty, 1)}$. In particular, this conclusion holds when $r=1+\epsilon$ for all $\epsilon>0$.

Proof. Let $r>1$. We proceed as in the proof of Proposition 4.4, and integrate

$$
\mathcal{V}_{\Psi} \sigma_{\mathrm{TH}}(x, \boldsymbol{t}, \boldsymbol{\xi}, \nu)
$$

in the following order:

$$
\begin{aligned}
& x \rightarrow r_{0}=\infty, \quad t_{1} \rightarrow r_{1}=1, \quad t_{2} \rightarrow r_{2}=r>1, \quad t_{3} \rightarrow r_{3}=r>1, \\
& \xi_{1} \rightarrow s_{1}=\infty, \quad \xi_{2} \rightarrow s_{2}=\infty, \quad \xi_{3} \rightarrow s_{3}=\infty, \quad \nu \rightarrow s_{0}=1 .
\end{aligned}
$$


In particular, we estimate

$$
\begin{aligned}
& \left\|\sigma_{\mathrm{TH}}\right\|_{\mathcal{M}^{(\infty, 1, r, r),(\infty, \infty, \infty, 1)}}=\int_{\mathbb{R}} d \nu \sup _{\xi_{1}, \xi_{2}, \xi_{3}}\left(\int_{\mathbb{R}} d t_{3} \int_{\mathbb{R}} d t_{2} \int_{\mathbb{R}} d t_{1} \sup _{x}\left|\mathcal{V}_{\Psi} \sigma_{H}(x, \boldsymbol{t}, \boldsymbol{\xi}, \nu)\right|^{r}\right)^{1 / r} \\
& =\int_{\mathbb{R}} d \nu \sup _{\xi_{1}, \xi_{2}, \xi_{3}}\left(\int_{\mathbb{R}} d t_{3} \int_{\mathbb{R}} d t_{2} \int_{\mathbb{R}} d t_{1} \sup _{x}|\hat{\psi}(\nu)|^{r}\left|\hat{\psi}\left(-t_{1}-t_{2}\right)\right|^{r}\right. \\
& \left.\left|\hat{\psi}\left(-2 t_{1}-t_{3}\right)\right|^{r}\left|V_{\psi} \sigma\left(\xi_{1}-\xi_{2}-2 \xi_{3},-t_{1}\right)\right|^{r}\right)^{1 / r} \\
& =\|\hat{\psi}\|_{1} \sup _{\xi_{1}, \xi_{2}, \xi_{3}}\left(\int_{\mathbb{R}} d t_{3} \int_{\mathbb{R}} d t_{2} \int_{\mathbb{R}} d t_{1}\left|\hat{\psi}\left(-t_{1}-t_{2}\right)\right|^{r}\left|\hat{\psi}\left(-2 t_{1}-t_{3}\right)\right|^{r}\right. \\
& \left.\left|V_{\psi} \sigma\left(\xi_{1}-\xi_{2}-2 \xi_{3},-t_{1}\right)\right|^{r}\right)^{1 / r} \\
& =\|\hat{\psi}\|_{1} \sup _{\xi_{1}, \xi_{2}, \xi_{3}}\left(\int_{\mathbb{R}} d t_{3} \int_{\mathbb{R}} d t_{2} \int_{\mathbb{R}} d t_{1}\left|\hat{\psi}\left(t_{2}+t_{1}\right)\right|^{r}\left|\hat{\psi}\left(t_{3}+2 t_{1}\right)\right|^{r}\right. \\
& \left.\left|V_{\psi} \sigma\left(\xi_{1}-\xi_{2}-2 \xi_{3},-t_{1}\right)\right|^{r}\right)^{1 / r} \\
& =\|\hat{\psi}\|_{1} \sup _{\xi_{1}, \xi_{2}, \xi_{3}}\left(\int_{\mathbb{R}} d t_{3} \int_{\mathbb{R}} d t_{2} \int_{\mathbb{R}} d t_{1}\left|\hat{\psi}\left(t_{2}-t_{1}\right)\right|^{r}\left|\hat{\psi}\left(2\left(t_{3}-t_{1}\right)\right)\right|^{r}\right. \\
& \left.\left|V_{\psi} \sigma\left(\xi_{1}-\xi_{2}-2 \xi_{3}, t_{1}\right)\right|^{r}\right)^{1 / r} \\
& =\|\hat{\psi}\|_{1} \sup _{\xi_{1}, \xi_{2}, \xi_{3}}\left(\int_{\mathbb{R}} d t_{3} \int_{\mathbb{R}} d t_{2} \int_{\mathbb{R}} d t_{1}\left|\hat{\psi}\left(t_{1}\right)\right|^{r}\left|\hat{\psi}\left(2\left(t_{2}-t_{3}-t_{1}\right)\right)\right|^{r}\right. \\
& \left.\left|V_{\psi} \sigma\left(\xi_{1}-\xi_{2}-2 \xi_{3}, t_{2}-t_{1}\right)\right|^{r}\right)^{1 / r} \\
& =\|\hat{\psi}\|_{1} \sup _{\xi_{1}, \xi_{2}, \xi_{3}}\left(\int_{\mathbb{R}} d t_{3} \int_{\mathbb{R}} d t_{2}|\hat{\psi}|^{r} *\left(\left|T_{t_{3}} \hat{\psi}_{2}\right|^{r}\left|\widetilde{V_{\psi} \sigma}\left(\xi_{1}-\xi_{2}-2 \xi_{3}, \cdot\right)\right|^{r}\right)\left(t_{2}\right)\right)^{1 / r}
\end{aligned}
$$

where $\hat{\psi}_{2}(\xi)=\hat{\psi}(2 \xi)$, and $\widetilde{V_{\psi} \sigma}\left(\xi_{1}-\xi_{2}-2 \xi_{3}, \eta\right)=V_{\psi} \sigma\left(\xi_{1}-\xi_{2}-2 \xi_{3},-\eta\right)$. Consequently,

$$
\begin{aligned}
\left\|\sigma_{\mathrm{TH}}\right\|_{\mathcal{M}^{(\infty, 1, r, r),(\infty, \infty, \infty, 1)}} & \left.\leq\|\hat{\psi}\|_{1}\|\hat{\psi}\|_{r} \sup _{\xi_{1}, \xi_{2}, \xi_{3}}\left(\int_{\mathbb{R}} d t_{3} \int_{\mathbb{R}} d t_{2}\left|\hat{\psi}_{2}\left(t_{2}-t_{3}\right)\right|^{r}\left|\widetilde{V_{\psi} \sigma}\left(\xi_{1}-\xi_{2}-2 \xi_{3}, t_{2}\right)\right|^{r}\right)\right)^{1 / r} \\
& =\|\hat{\psi}\|_{1}\|\hat{\psi}\|_{r} \sup _{\xi_{1}, \xi_{2}, \xi_{3}}\left(\int_{\mathbb{R}} d t_{3}\left|\hat{\psi}_{2}\right|^{r} *\left|\widetilde{V_{\psi} \sigma}\left(\xi_{1}-\xi_{2}-2 \xi_{3}, \cdot\right)\right|^{r}\left(t_{3}\right)\right)^{1 / r} \\
& \leq\|\hat{\psi}\|_{1}\|\hat{\psi}\|_{r}\left\|\hat{\psi}_{2}\right\|_{r} \sup _{\xi_{1}, \xi_{2}, \xi_{3}}\left(\int_{\mathbb{R}} d t_{3}\left|\widetilde{V_{\psi} \sigma}\left(\xi_{1}-\xi_{2}-2 \xi_{3}, t_{3}\right)\right|^{r}\right)^{1 / r} \\
& =\|\hat{\psi}\|_{1}\|\hat{\psi}\|_{r}\left\|\hat{\psi}_{2}\right\|_{r} \sup _{\xi_{1}, \xi_{2}, \xi_{3}}\left(\int_{\mathbb{R}} d t_{3}\left|V_{\psi} \sigma\left(\xi_{1}-\xi_{2}-2 \xi_{3}, t_{3}\right)\right|^{r}\right)^{1 / r}
\end{aligned}
$$


The proof is complete by observing that the proof of Proposition 4.4 implies that

$$
\sup _{\xi_{1}, \xi_{2}, \xi_{3}}\left(\int_{\mathbb{R}} d t_{3}\left|V_{\psi} \sigma\left(\xi_{1}-\xi_{2}-2 \xi_{3}, t_{3}\right)\right|^{r}\right)^{1 / r}<\infty .
$$

Using this result and Theorem 3.4 for $m=3$ we can give the following initial result on the boundedness of $\mathrm{TH}$ on product of modulation spaces.

Theorem 4.8. For $p, p_{0}, p_{1} \in(1, \infty)$ and $1 \leq q \leq \infty$, the trilinear Hilbert transform $T H$ is bounded from $M^{p_{1}, 1} \times M^{p, q} \times M^{p^{\prime}, q^{\prime}}$ into $M^{p_{0}, \infty}$ and we have the following estimate:

$$
\|T H(f, g, h)\|_{M^{p_{0}, \infty}} \leq C\|f\|_{M^{p_{1}, 1}}\|g\|_{M^{p, q}}\|h\|_{M^{p^{\prime}, q^{\prime}}}
$$

for all $f, g, h \in S(\mathbb{R})$, where the constant $C>0$ is independent of $f, g, h$.

Remark 4.9. Before proving this result we point out that the strongest results are obtained by choosing $p_{0}$ as close to 1 as possible and $p_{1}$ as close to $\infty$ as possible.

As special case, we see that TH boundedly maps

$$
M^{r, 1} \times L^{2} \times L^{2} \longrightarrow M^{1+\epsilon, \infty}
$$

for every $r<\infty$ and $\epsilon>0$.

Proof. We set $r=\min \left\{p_{0}, p_{1}^{\prime}, p, p^{\prime}\right\}>1$. The symbol of $\mathrm{TH}$ is in the symbol modulation space with decay parameters $r_{0}=\infty, r_{1}=1, r_{2}=r_{3}=r>1$ as used in Theorem 3.4. Note that here, $\kappa$ is the identity permutation, so $z=0$. The boundedness conditions in Theorem 3.4 now read

$k=0: \quad 0+\frac{1}{\widetilde{p}_{1}} \leq 1 ;$

$k=1: \quad 0+\frac{1}{\widetilde{p}_{2}}+\frac{1}{\widetilde{p}_{1}}+1 \leq 2 ;$

$k=2: \quad 0+\frac{1}{\widetilde{p}_{3}}+\frac{1}{\widetilde{p}_{1}}+1+\frac{1}{\widetilde{p}_{2}}+\frac{1}{r} \leq 3 ;$

$k=3: \quad 0+\frac{1}{\widetilde{p}_{1}}+1+\frac{1}{\widetilde{p}_{2}}+\frac{1}{r}+\frac{1}{\widetilde{p}_{3}}+\frac{1}{r} \geq 3+\frac{1}{p_{0}} ;$

where

$$
p_{1} \leq \widetilde{p}_{1} \leq r_{1}^{\prime}=\infty, \quad p_{2} \leq \widetilde{p}_{2} \leq r^{\prime}, \quad p_{3} \leq \widetilde{p}_{3} \leq r^{\prime} .
$$

The four conditions above reduce to

$k=1: \quad \frac{1}{\widetilde{p}_{1}}+\frac{1}{\widetilde{p}_{2}} \leq 1 ;$

$k=2: \quad \frac{1}{\widetilde{p}_{1}}+\frac{1}{\widetilde{p}_{2}}+\frac{1}{\widetilde{p}_{3}} \leq 2-\frac{1}{r} ;$

$k=3: \quad \frac{1}{\widetilde{p}_{1}}+\frac{1}{\widetilde{p}_{2}}+\frac{1}{\widetilde{p}_{3}} \geq 2-\frac{2}{r}+\frac{1}{p_{0}} ;$

For simplicity, we now set $p_{2}=\widetilde{p}_{2}=p_{3}^{\prime}=\widetilde{p}_{3}^{\prime} \in\left[r, r^{\prime}\right]$ and obtain

$k=1: \quad \frac{1}{\widetilde{p}_{1}} \leq \frac{1}{\widetilde{p}_{3}} ;$

$k=2: \quad \frac{1}{\widetilde{p}_{1}} \leq 1-\frac{1}{r}$;

$k=3: \quad \frac{1}{\widetilde{p}_{1}} \geq 1-\frac{2}{r}+\frac{1}{p_{0}} ;$

that is

$k=1: \quad \widetilde{p}_{1} \geq \widetilde{p}_{3} ;$

$k=2: \quad \widetilde{p}_{1} \geq r^{\prime} ;$

$k=3: \quad \frac{1}{\widetilde{p}_{1}} \geq 1-\frac{2}{r}+\frac{1}{p_{0}}$ 
Note that the condition for $k=1$ follows from the $k=2$ condition since $p_{3}^{\prime} \leq r^{\prime}$.

For the existence of $\widetilde{p}_{1} \geq p_{1}$, satisfying the $k=2$ and $k=3$ conditions, we require $2-\frac{1}{r} \geq$ $2-\frac{2}{r}+\frac{1}{p_{0}}$, which is $r \leq p_{0}$, a condition that is met. Some $\widetilde{p}_{1} \geq p_{1}$ will satisfy all conditions if $\frac{1}{p_{1}} \geq 1-\frac{2}{r}+\frac{1}{p_{0}}$. Indeed,

$$
1-\frac{2}{r}+\frac{1}{p_{0}}=1-\frac{1}{r}+\frac{1}{p_{0}}-\frac{1}{r} \leq 1-\frac{1}{r} \leq \frac{1}{p_{1}} .
$$

We now consider the conditions of Theorem 3.4 on the frequency side. We choose $\rho$ to be the identity permutation on $\{1,2,3,4\}, s_{1}=s_{2}=s_{3}=\infty, s_{4}=1$. We now have to consider existence of $\widetilde{q}_{1} \geq s_{1}^{\prime}=1, \widetilde{q}_{2} \geq s_{2}^{\prime}=1$, and $\widetilde{q}_{3} \geq s_{3}^{\prime}=1$ with

$$
\begin{array}{ll}
k=1: & \frac{1}{\widetilde{q}_{1}}+\frac{1}{\widetilde{q}_{2}}+\frac{1}{\widetilde{q}_{3}} \geq 2 ; \\
k=2: & \frac{1}{\widetilde{q}_{2}}+\frac{1}{\widetilde{q}_{3}} \geq 1 ; \\
k=3: & \frac{1}{\widetilde{q}_{3}} \geq 0 ; \\
k=4: & \frac{1}{\widetilde{q}_{1}}+\frac{1}{\widetilde{q}_{2}}+\frac{1}{\widetilde{q}_{3}} \geq 2+\frac{1}{q_{4}} .
\end{array}
$$

These conditions reduce to

$$
\frac{1}{\widetilde{q}_{2}}+\frac{1}{\widetilde{q}_{3}} \geq 1, \quad \frac{1}{\widetilde{q}_{1}}+\frac{1}{\widetilde{q}_{2}}+\frac{1}{\widetilde{q}_{3}} \geq 2+\frac{1}{q_{4}} .
$$

To assume optimally large $q_{1}, q_{2}, q_{3}$, we choose $\tilde{q}_{2}=q_{2}=q, q_{3}^{\prime}=\tilde{q}_{3}^{\prime}=q^{\prime}$ and $\frac{1}{q_{1}}=1+\frac{1}{q_{4}}$, the latter only being satisfied if $q_{1}=1$ and $q_{4}=\infty$.

In [50, Theorem 13] it is proved that the trilinear Hilbert transform is bounded from $L^{p} \times L^{q} \times \mathcal{A}$ into $L^{r}$ whenever $1<p, q \leq \infty, 2 / 3<r<\infty$ and $\frac{1}{p}+\frac{1}{q}=\frac{1}{r}$, where $\mathcal{A}$ is the Fourier algebra. In particular, for $p=q=2$, then $r=1$ and the operator maps boundedly $L^{2} \times L^{2} \times \mathcal{A}$ into $L^{1}$.

From [60, Proposition 1.7] we know that when $p \in(1,2)$ and $p<q^{\prime}<p^{\prime}$, then $\mathcal{F} L^{q^{\prime}} \subset M^{p^{\prime}, q^{\prime}}$. We can then conclude that TH continuously maps $M^{p_{1}, 1} \times M^{p, q} \times \mathcal{F} L^{q^{\prime}}$ into $M^{p_{0}, \infty}$.

\section{Acknowledgment}

K. A. Okoudjou was partially supported by a RASA from the Graduate School of UMCP, the Alexander von Humboldt foundation, and by a grant from the Simons Foundation (\#319197 to Kasso Okoudjou). G. E. Pfander appreciates the hospitality of the mathematics departments at MIT and at the TU Munich. This project originated during a sabbatical at MIT and was completed during a visit of TU Munich as John von Neumann Visiting Professor. G. E. Pfander also appreciates funding from the German Science Foundation (DFG) within the project Sampling of Operators.

\section{REFERENCES}

[1] A. Benedek and R. Panzone, The Space $L^{p}$, with Mixed Norm, Duke Math. J. 28 (1961), 301-324.

[2] Á. Bényi and R. Torres, Almost orthogonality and a class of bounded bilinear pseudodifferential operators, Math. Res. Lett. 11 (2004), no. 1, 1-11. 
[3] Á. Bényi, N. Tzirakis, Multilinear almost diagonal estimates and applications, Studia Math. 164 (2004), no. $1,75-89$.

[4] Á. Bényi, L. Grafakos, K. Gröchenig, K. Okoudjou, A class of Fourier multipliers for modulation spaces, Appl. Comput. Harmon. Anal. 19 (2005), no. 1, 131-139.

[5] Á. Bényi, K. Gröchenig, K. Okoudjou, L. Rogers, Unimodular Fourier multipliers for modulation spaces, J. Funct. Anal. 246 (2007), no. 2, 366-384.

[6] A. Bényi and K. Okoudjou, Bilinear pseudodifferential operators on modulation spaces, J. Fourier Anal. Appl. 10 (2004), no. 3, 301-313.

[7] A. Bényi, K. Gröchenig, C. Heil and K. Okoudjou, Modulation spaces and a class of bounded multilinear pseudodifferential operators, J. Operator Theory 54 (2005), no. 2, 387-399.

[8] A. Bényi and K. A. Okoudjou, Modulation space estimates for multilinear pseudodifferential operators, Studia Math. 172 (2006), no. 2, 169-180.

[9] A. Bényi and K. A. Okoudjou, Local well-posedness of nonlinear dispersive equations on modulation spaces, Bull. Lond. Math. Soc. 41 (2009), no. 3, 549-558.

[10] S. Bishop, Mixed modulation spaces and their application to pseudodifferential operators, J. Math. Anal. Appl. 363 (2010) 1, 255-264.

[11] A. P. Calderón, R. Vaillancourt, On the boundedness of pseudo-differential operators, J. Math. Soc. Japan, 23 (1971) 374-378.

[12] V. Catană, S. Molahajloo and M. W. Wong, $L^{p}$-Boundedness of Multilinear Pseudo-Differential Operators, in Pseudo-Differential Operators: Complex Analysis and Partial Differential Equations Operator Theory: Advances and Applications 205, Birkhäuser, 2010, 167-180.

[13] L. Carleson, On convergence and growth of partial sums of Fourier series, Acta Math. 116 (1966), $135-157$.

[14] E. Codero and F. Nicola, Metaplectic Representation on Wiener Amalgam Spaces and Applications to the Schrödinger Equation, J. Funct. Anal. 254 (2008), 506-534.

[15] E. Codero and F. Nicola, Pseudodifferential Operators on $L^{p}$, Wiener Amalgam and Modulation Spaces, Int. Math. Res. Notices 10 (2010), 1860-1893.

[16] F. Concetti, J, Toft, Trace Ideals for Fourier Integral Operators with Non-Smooth Symbols, in PseudoDifferential Operators: Partial Differential Equations and Time Frequency Analysis, Fields Institute Communications, 52 (2007), 255-264.

[17] R. R. Coifman, Y. Meyer, Yves, "Au delà des opérateurs pseudo-différentiels," Astérisque, 57, Société Mathématique de France, Paris, 1978.

[18] W. Czaja, Boundedness of Pseudodifferential Operators on Modulation Spaces, J. Math. Anal. Appl. 284 (1) (2003), 389-396.

[19] C. Fefferman, Pointwise convergence of Fourier series, Ann. of Math. 98 (1973), 551-571.

[20] H. G. Feichtinger, Modulation spaces on locally Abelian groups, Technical report, University of Vienna, 1983. Updated version appeared in: Proceedings of International Conference on Wavelets and Applications 2002, Allied Publishers, Chennai, India, 2003, pp. 99-140.

[21] H. G. Feichtinger, Atomic Characterization of Modulation Spaces through the Gabor-Type Representations, Rocky Mountain J. Math. 19 (1989), 113-126.

[22] H. G. Feichtinger, On a New Segal Algebra, Monatsh. Math. 92 (1981), 269-289.

[23] H. G. Feichtinger and K. Gröchenig, Banach Spaces Related to Integrable Group Representations and Their Atomic Decompositions I, J. Funct. Anal. 86 (1989), 307-340.

[24] H. G. Feichtinger and K. Gröchenig, Banach Spaces Related to Integrable Group Representations and Their Atomic Decompositions II, Monatsh. Math. 108 (1989), 129-148.

[25] H. G. Feichtinger and K. Gröchenig, Gabor Wavelets and the Heisenberg Group: Gabor Expansions and Short Time Fourier Transform from the Group Theoretical Point of View, in Wavelets: a tutorial in theory and applications, Academic Press, Boston, 1992.

[26] H. G. Feichtinger and K. Gröchenig, Gabor Frames and Time-Frequency Analysis of Distributions, J. Funct. Anal. 146 (1997), 464-495.

[27] H. G. Feichtinger, F. Weisz, Wiener amalgams and pointwise summability of Fourier transforms and Fourier series, Math. Proc. Cambridge Philos. Soc. 140 (2006), no. 3, 509-536.

[28] H. G. Feichtinger, G. Narimani, Fourier multipliers of classical modulation spaces, Appl. Comput. Harmon. Anal. 21 (2006), no. 3, 349-359.

[29] H. G. Feichtinger, F. Weisz, Gabor analysis on Wiener amalgams, Sampl. Theory Signal Image Process. 6 (2007), no. 2, 129-150. 
[30] G. B. Folland, "Harmonic analysis in phase space," Annals of Mathematics Studies, 122, Princeton University Press, Princeton, NJ, (1989).

[31] L. Grafakos, C. Lennard, Characterization of $L^{p}\left(\mathbb{R}^{n}\right)$ using Gabor frames, J. Fourier Anal. Appl. 7 (2001), no. $2,101-126$.

[32] L. Grafakos, R. H. Torres, Rodolfo A multilinear Schur test and multiplier operators, J. Funct. Anal. 187 (2001), no. 1, 1-24.

[33] K. Gröchenig, C. Heil, Gabor meets Littlewood-Paley: Gabor expansions in $L^{p}\left(\mathbb{R}^{d}\right)$, Studia Math. 146 (2001), no. 1, 15-33.

[34] K. Gröchenig, Foundation of Time-Frequency Analysis, Brikhäuser, Boston, 2001.

[35] K. Gröchenig and C. Heil, Counterexamples for Boundedness of Pseudodifferential Operators, Osaka J. Math. 41 (3) (2004), 681-691.

[36] K. Gröchenig and C. Heil, Modulation Spaces and Pseudodifferential Operators, Integr. Equat. Oper. th. 34 (4) (1999), 439-457.

[37] C. Heil, J. Ramanathan, P. Topiwala, Singular values of compact pseudodifferential operators, J. Funct. Anal. 150 (1997), no. 2, 426-452.

[38] Y. M. Hong and G. E. Pfander, Irregular and multi-channel sampling of operators, 2009, Preprint.

[39] L. Hörmander, The Analysis of Linear Partial Differential Operators I, Second Edition, Springer-Verlag, Berlin, 1990.

[40] L. Hörmander, The Weyl Calculus of Pseudodifferential Operators, Comm. Pure Appl. Math. 32 (1979), 360-444.

[41] I. L. Hwang and R. B. Lee, $L^{p}$-Boundedness of Pseudo-Differential Operators of Class $S_{0,0}$, Trans. Amer. Math. Soc. 346 (2) (1994), 489-510.

[42] H. Kumano-Go, Pseudo-Differential Operators, Translated by Hitoshi Kumano-Go, Rémi Vaillancourt and Michihiro Nagase, MIT Press, 1982.

[43] M. T. Lacey, On the bilinear Hilbert transform, Proceedings of the International Congress of Mathematicians, Vol. II (Berlin, 1998). Doc. Math. 1998, Extra Vol. II, 647-656.

[44] M. T. Lacey, C. Thiele, $L^{p}$ estimates on the bilinear Hilbert transform for $2<p<\infty$, Ann. of Math. (2) 146 (1997), no. 3, 693-724.

[45] M. Lacey, C. Thiele, On Calderón's conjecture, Ann. of Math. (2) 149 (1999), no. 2, 475-496.

[46] M. Lacey, C. Thiele, Aproof of boundedness of the Carleson operator, Math. Res. Lett. 7 (2000), no. 4, 361-370.

[47] G. F. Margrave, M. P. Lamoureux, J. P. Grossman, D. C. Henley and V. Iliescu, The Gabor transform, pseudodifferential operators, and seismic deconvolution, Integrated Computer-Aided Engineering, 12 (2005), no. 1, 43-55.

[48] Y. Meyer, R. Coifman,, "Wavelets," Calderón-Zygmund and multilinear operators. Translated from the 1990 and 1991 French originals by David Salinger. Cambridge Studies in Advanced Mathematics, 48, Cambridge University Press, Cambridge, 1997.

[49] S. Molahajloo, G. E. Pfander, Boundedness of pseudo-differential operators on $L^{p}$, Sobolev and modulation spaces, Math. Model. Nat. Phenom. 8 (2013), no. 1, 175-192.

[50] C. Muscalu, T. Tao, C. Thiele, Multi-linear operators given by singular multipliers, J. Amer. Math. Soc., 15 (2002), no. 2, 469-496.

[51] K. A. Okoudjou, A Beurling-Helson Type Theorem for Modulation Spaces, J. Func. Spaces Appl., 7 (1) (2009), 33-41.

[52] G. E. Pfander and D. Walnut, Operator Identification and Feichtinger's Algebra, Sampl. Theory Signal Image Process. 5 (2) (2006), 151-168.

[53] G. E. Pfander, Sampling of Operators, arxiv: 1010.6165.

[54] R. Rochberg and K. Tachizawa, Pseudodifferential operators, Gabor frames, and local trigonometric bases, in Gabor Analysis and Algorithms: Theory and Applications (H. G. Feichtinger and T. Strohmer, eds.), Birkhäuser, Boston, 1997, pp. 171-192.

[55] J. Sjöstrand, An Algebra of Pseudodifferential Operators, Math. Res. Lett. 1 (2) (1994), 185-192.

[56] J. Sjöstrand, Wiender Type Algebras of Pseudodifferential Operators, in Séminaire Équations aux dérivées Partielles, 1994-1995, exp. 4, 1-19.

[57] E. M. Stein, "Harmonic analysis: real-variable methods, orthogonality, and oscillatory integrals," Princeton University Press, Princeton, N.J., (1993).

[58] T. Strohmer, Pseudodifferential operators and Banach algebras in mobile communications, Appl. Comput. Harmon. Anal. 20 (2006), no. 2, 237-249. 
[59] M. E. Taylor, "Pseudodifferential operators," Princeton University Press, Princeton, N.J., (1981).

[60] J. Toft, Continuity Properties for Modulation Spaces, with Applications to Pseudo-Differential Calculus I, J. Funct. Anal. 207 (2004), 399-429

[61] J. Toft, Continuity Properties for Modulation Spaces, with Applications to Pseudo-Differential Calculus II, Ann. Glob. Anal. Geom. 26 (2004), 73-106.

[62] J. Toft, Fourier Modulation Spaces and Positivity in Twisted Convolution Algebra, Integral Transforms and Special Functions 17 nos. 2-3 (2006), 193-198.

[63] J. Toft, Pseudo-Differential Operators with Smooth Symbols on Modulation Spaces, CUBO. 11 (2009), $87-107$.

[64] J. Toft, S. Pilipovic, N. Teofanov, (2010). Micro-Local Analysis in Fourier Lebesgue and Modulation Spaces. Part II, J. Pseudo-Differ. Oper. Appl. 1 (2010), 341-376.

[65] J. Toft, itContinuity and Schatten properties for pseudo-differential operators on modulation spaces,in "Modern trends in pseudo-differential operators", Oper. Theory Adv. Appl., Birkhäuser, 172 (2007), 173-206, Basel.

[66] K. Tachizawa, The boundedness of pseudodifferential operators on modulation spaces, Math. Nachr. 168 (1994), 263-277.

[67] M. W. Wong, An Introduction to Pseudo-Differential Operators, Second Edition, World Scientific, 1999.

[68] M. W. Wong, Fredholm Pseudo-Differential Operators on Weighted Sobolev Spaces, Ark. Mat. 21 (2) (1983), $271-282$.

[69] M. W. Wong, Weyl Transforms, Springer-Verlag, 1998.

Shahla Molahajloo, Department of Mathematics, Institute for Advanced Studies in Basic SciENCES (IASBS), P. O. Box 45195-1159, Gava ZANG, ZANJAn 45137-66731 IrAn

E-mail address: Molahajloo@iasbs.ac.ir

Kasso A. Okoudjou, Department of Mathematics, University of Maryland, College Park, MD, 20742 USA

E-mail address: kasso@math.umd.edu

Götz E. Pfander, School of Science and Engineering, Jacobs University, 28759 Bremen, Germany

E-mail address: g.pfander@jacobs-university.de 